\title{
Damage Identification using Inverse Methods
}

\author{
Michael I. Friswell \\ Department of Aerospace Engineering, University of Bristol, Bristol BS8 1TR, UK. \\ m.i.friswell@bristol.ac.uk
}

\begin{abstract}
This chapter gives an overview of the use of inverse methods in damage detection and location, using measured vibration data. Inverse problems require the use of a model and the identification of uncertain parameters of this model. Damage is often local in nature and although the effect of the loss of stiffness may require only a small number of parameters, the lack of knowledge of the location means that a large number of candidate parameters must be included. This leads to potential ill-conditioning problems, and this topic is reviewed in this chapter. This chapter then goes on to discuss a number of problems that exist with the inverse approach to structural health monitoring, including modelling errors, environmental effects, damage localisation, regularisation, models of damage and sensor validation.
\end{abstract}

\section{Introduction to Inverse Methods}

Inverse methods combine an initial model of the structure and measured data to improve the model or test an hypothesis. In practice the model is based on finite element analysis and the measurements are acceleration and force data, often in the form of a modal database, although frequency response function (FRF) data may also be used. The estimation techniques are often based on the methods of model updating, which have had some success in improving models and understanding the underlying dynamics, especially for joints (Friswell and Mottershead, 1995; Mottershead and Friswell, 1993). Model updating methods may be classified as sensitivity or direct methods. Sensitivity type methods rely on a parametric model of the structure and the minimisation of some penalty function based on the error between the measured data and the predictions from the model. These methods offer a wide range of parameters to update that have physical meaning and allow a degree of control over the optimisation process. The alternative is direct updating methods that change complete mass and/or stiffness matrices, although the updated models obtained are often difficult to interpret for health monitoring applications. These methods will be considered in more detail later. However it should be emphasised that a huge number of papers have been written on the application of inverse methods to damage identification, and this chapter aims to give an overview of the approaches rather than a complete literature review. This chapter will also consider some of the difficulties that occur when inverse methods are used for damage identification (Friswell, 2007; Doebling et al., 1998).

The four stages of damage estimation, first given by Rytter (1993), are now well established as detection, location, quantification and prognosis. Detection is readily 
performed by pattern recognition methods or novelty detection (Worden, 1997; Worden et al., 2000). The key issue for inverse methods is location, which is equivalent to error localisation in model updating. Once the damage is located, it may be parameterised with a limited set of parameters and quantification, in terms of the local change in stiffness, is readily estimated. Prognosis requires that the underlying damage mechanism is determined, which may be possible using inverse methods using hypothesis testing among several candidate mechanisms. This questions is considered in more detail later in the chapter. However, once the damage mechanism is determined, the associated model is available for prognosis, and this is a great advantage of model based inverse methods.

\subsection{Objective Functions}

Friswell and Mottershead (1995) discussed sensitivity based methods in detail. The approach minimises the difference between modal quantities (usually natural frequencies and less often mode shapes) of the measured data and model predictions. This problem may be expressed as the minimization of $J$, where

$$
J(\theta)=\left\|\mathbf{z}_{m}-\mathbf{z}(\theta)\right\|^{2}=\epsilon^{T} \epsilon
$$

and

$$
\epsilon=\mathbf{z}_{m}-\mathbf{z}(\theta) .
$$

Here $\mathbf{z}_{m}$ and $\mathbf{z}(\theta)$ are the measured and computed modal vectors, $\theta$ is a vector of all unknown parameters, and $\epsilon$ is the modal residual vector. The modal vectors may consist of both natural frequencies and mode shapes, although often mode shapes are only used to pair individual modes. If mode shapes are included then they must be carefully normalised, the sensor locations must be carefuly matched to the finite element degrees of freedom and weighting should be applied to Equation (1.1).

Frequency response functions may also be used, although a model of damping is required, and the penalty function is often a very complicated function of the parameters with many local minima, making the optimisation very difficult. dos Santos et al. (2005) presents an example of such a method for damage in a composite structure.

\subsection{Sensitivity Methods}

Sensitivity based methods allow a wide choice of physically meaningful parameters and these advantages has led to their widespread use in model updating. The approach is very general and relies on minimising a penalty function, which usually consists of the error between the measured quantities and the corresponding predictions from the model. Parameters are then chosen that are assumed uncertain, and these are usually estimated by approximating the penalty function using a truncated Taylor series and iterating to obtain a converged solution. If there are sufficient measurements and a restricted set of parameters then the identification may be well-conditioned. Often some form of regularisation must be applied, and this is considered in detail later. Other optimisation methods may be used, such as quadratic programming, simulated annealing or genetic algorithms, but these are not considered further in this chapter. Problems will also arise 
if an incorrect or incomplete set of parameters is chosen, or even worse, if the structure of the model is wrong.

The modal residual in Equation (1.1) is a non-linear function of the parameters and the minimization is solved using a truncated linear Taylor series and iteration. Thus the Taylor series is

$$
\mathbf{z}_{m}=\mathbf{z}_{j}+\mathbf{S}_{j} \delta \theta_{j}+\text { higher order terms }
$$

where

$$
\mathbf{z}_{j}=\mathbf{z}\left(\theta_{j}\right), \quad \mathbf{S}_{j}=\mathbf{S}\left(\theta_{j}\right), \quad \delta \theta_{j}=\theta_{m}-\theta_{j}
$$

The matrix $\mathbf{S}_{j}$ consists of the first derivatives of the modal quantities with respect to the model parameters, index $j$ denotes the $j$ th iteration and $\theta_{m}$ is the parameter vector that gives the measured outputs. Standard methods exist to calculate the modal derivatives required (Friswell and Mottershead, 1995; Adhikari and Friswell, 2001). By neglecting higher order terms in Equation (1.3), an iterative scheme may be derived, using the linear approximation,

$$
\delta \mathbf{z}_{j}=\mathbf{S}_{j} \delta \theta_{j}
$$

where $\delta \mathbf{z}_{j}=\mathbf{z}_{m}-\mathbf{z}_{j}$ and $\delta \theta_{j}=\theta_{j+1}-\theta_{j}$. Often, for damage location studies, only the residual and sensitivity matrix for the initial model are used. Avoiding iteration reduces the computation required, particularly where multiple parameter sets have to be estimated. However, particularly if the damage is severe, there is a risk that the wrong location is identified.

As indicated above, one of the problems with sensitivity methods is the need for a parameteric model of the damage. Mottershead et al. (1999) proposed an approach where the system was constrained so that unknown stiffnesses are replaced with rigid connections. The constraint is not imposed physically but the behaviour inferred from the unconstrained measurements. The best fit between the measured and predicted data is obtained when the damage is located in the substructure that is made rigid.

\subsection{Model Parameters}

One of the key aspects of a model based identification method is the parameterisation of the candidate damage. Since inverse approaches rely on a model of the damage, the success of the estimation is dependent on the quality of the model used. The type of model used will depend on the type of structure and the damage mechanism, which leads to an increase either in local or distributed flexibility. The damage model may be simple or complex. For example, a cracked beam may be modelled as a reduction in stiffness in a large finite element or substructure, or alternatively using a very detailed model from fracture mechanics. Whether such a detailed model is justified will often depend on the requirements of the estimation procedure and the quality of the measured data. Using a measured modal model consisting of the lower natural frequencies and associated mode shapes will mean that only a coarse model of the damage may be identified. The simple example used for illustration will use element stiffnesses as the parameters and is the simplest form of equivalent model for the damage. More detailed models of damage will be considered in Section 3 . 


\subsection{Optimisation Procedures and Ill-Conditioning}

When the parameters of a model are unknown, they must be estimated using measured data. Usually the measured response will be a non-linear function of the parameters. In these cases, minimizing the error between the measured and predicted response will produce a non-linear optimisation problem, with the usual questions about convergence and local minima. The most common approach is to linearise the residuals, obtain a least squares solution and iterate. If the identification problem is well posed then this simple approach will be adequate. The usual response to problems encountered in the optimisation is to try more advanced algorithms, but often the issue is that the estimation problem has not been posed correctly, and including some physical insight into the problem provides a much better solution.

Probably the most important difficulty in parameter estimation is ill-conditioning. In the worst case this can mean that there is no unique solution to the estimation problem, and many sets of parameters are able to fit the data. Many optimization procedures result in the solution of linear equations for the unknown parameters. The use of the singular value decomposition (SVD) (Golub and van Loan, 1996) for these linear equations enables ill-conditioning to be identified and quantified. The options are then to increase the available data, which is often difficult and costly, or to provide extra conditions on the parameters. These can take the form of smoothness conditions (for example, the truncated SVD), minimum norm parameter values (Tikhonov regularization) or minimum changes from the initial estimates of the parameters (Hansen, 1992, 1994).

Black box methods are often not considered as model based approaches. However any simulation of an input-output relationship must make some assumptions about the underlying process, and hence essentially has an underlying model. For example a neural network is essentially a very sophisticated curve fitting algorithm, and ill-conditioning is a major problem, evidenced by over-fitting and a lack of generalisation. The advantage of neural networks is that the class of input-output relationships that may be fitted is huge. However, better results will always be obtained if physical insight is used to guide the modelling and estimation process. Indeed there is often a need to reduce the number of input nodes to present to a neural network, and understanding is vital to obtain the correct feature extraction and data reduction. Another use of physical models is the generation of training or test data for these identification schemes. Typically experimental data for a sufficient range of events is difficult or expensive to obtain. Since running a model many times is relatively easy and cheap, these simulations may be used to increase the quantity of the test data. However it is vital that this simulated data correctly reproduces the important features of the real structure, and hence requires a validated and, if necessary, updated model.

Neural networks and genetic algorithms have been viewed as potential saviours for the solution of the difficult problems in damage location. Although these methods may be useful in some circumstances they do not deal with the root cause of the problem. Genetic algorithms have some advantage in finding a global minimum in very difficult optimisation problems, particularly where there are many local minima as is often the case in damage location. That said, the method still requires that the dynamics of the structure changes sufficiently and predictably enough for the optimisation to be 
meaningful. The crucial decision and difficulty is what to optimise, not the optimisation method used.

Neural networks are able to treat damage mechanisms implicitly, so that it is not necessary to model the structure in so much detail. The method can also deal with non-linear damage mechanisms easily. Models are still required to provide the training cases for the networks, and this is their major problem. There will always be systematic errors between the model used for training and the actual structure. For success, neural networks require that the essential features in the damaged structure were represented in the training data. The robustness of networks to these errors has not been tested sufficiently. Of course, the other major problem with both genetic algorithms and neural networks is that they require a huge amount of computation for structures of practical complexity, although these methods are well suited to parallel computation.

\subsection{Problems and Errors in Damage Identification}

The discussion thus far has indicated some of the problems with damage identification. There are always errors in the measured data and the numerical model that affect all of the algorithms. These errors, and the adequacy of the data, are now discussed. Damage identification algorithms should always be tested on realistic experimental examples, as many methods that work well on simulated data often fail due to the problems highlighted in this section. As a first step, methods may be tested using simulated data, but even then realistic systematic errors should be incorporated.

Modelling errors One of the major problems in damage location is the reliance on the finite element model. This model is also an important strength because the very incomplete set of measured data requires extra information from the model to be able to identify damage location. There will undoubtedly be errors even in the model of the undamaged structure. Thus if the measurements on the damaged structure are used to identify damage locations, the methods will have great difficulty in distinguishing between the actual damage sites and the location of errors in the original model. If suitable parameters are not included to allow for the undamaged model errors then the result will be a systematic error between the model and the data. Identification schemes generally have considerable difficulty with systematic errors. It is very likely that the original errors in the model will produce frequency changes that are far greater than those produced by the damage. There are two basic approaches to reducing this problem, although both rely on having measured data from an undamaged structure. The first is to update the finite element model of the undamaged structure to produce a reliable model (Friswell and Mottershead, 1995). Obviously the quality of the damage location assessment is critically dependent upon the updated model being physically meaningful (Friswell et al., 2001; Link and Friswell, 2003). Generally, this requires model validation using a control set of data not used for the updating. The second alternative uses differences between the damaged and undamaged response data in the damage location algorithm (Parloo et al., 2003; Titurus et al., 2003b). To first order, any error in the undamaged model of the structure that is also present in the damaged structure will be removed. This does rely on the structure remaining unchanged, except for the damage, 
between the two sets of measurements.

Another potential source of error is the mismach between the measurement locations and the model degrees of freedom. Such a mismatch makes the direct comparison of frequency response functions and mode shapes impossible, and the generation of residuals, inaccurate. The magnitude of the errors involved will depend on the mesh density in the sensor region and the complexity of the mode shapes. The best solution is to ensure nodes in the model exist at the sensor locations. Alternatively interpolation techniques may be used.

Environmental and other non-stationary effects One very difficult aspect of damage assessment is the change in the measured data due to environmental effects. This is one undesirable non-stationary effect and makes damage location very difficult. Of course progressive damage is also a non-stationary phenomenon, and damage can be difficult to identify if other non-stationary effects are also present. Typical environmental effects are demonstrated by highway bridges, especially those constructed using concrete, which have been the subject of many studies in damage location. For example, temperature changes can cause the stiffness properties of a bridge to change significantly, and the difficulty is to predict the effects of temperature from readily available measurements. Peeters and de Roeck (2001) reported on measurements of the Z24 bridge over a whole year and suggested a black box model to predict the temperature variation. Sohn et al. (1999) considered the effect of temperature on the Alamosa Canyon Bridge. Sohn et al. (2002) used a combination of time series analysis, neural networks and statistical inference to determine damage state for structures affected by the environmental conditions. Mickens et al. (2003) corrected frequency response function measurements by assuming the temperature affected the global stiffness of the structure. On a highway bridge, the changing traffic conditions cause different mass loading effects that can change the natural frequencies by as much as $1 \%$ (Zhang et al., 2002). There are further difficulties with highway bridges because they are highly damped with low natural frequencies. They are in a noisy environment and are difficult to excite. The frequency resolution in the measurements is invariably quite low, leading to considerable difficulties in detecting small frequency changes due to damage.

Typical of environmental effects are those in highway bridges. These bridges have been the subject of many studies in damage location, but in the UK, where most bridges are constructed using concrete, such identification has considerable problems with changes due to environmental factors (Wood, 1992). For example, concrete absorbs considerable moisture during damp weather, which considerably increases the mass of the bridge. Temperature changes the stiffness properties of the road surface, known as the black-top, significantly. On a hot summer's day in the UK, the road surface will provide little stiffness, but on a cold winter's day the stiffness contribution is considerable. The difficulty is trying to predict the effects of temperature and moisture absorption from readily available measurements. Figure 1 shows the variation of the first 4 natural frequencies of a concrete highway bridge in Birmingham, UK with soffit temperature (Wood, 1992). Soffit temperature is the variable that correlates best with the frequencies, but even then relatively large, unexplained variations in frequency occur. 


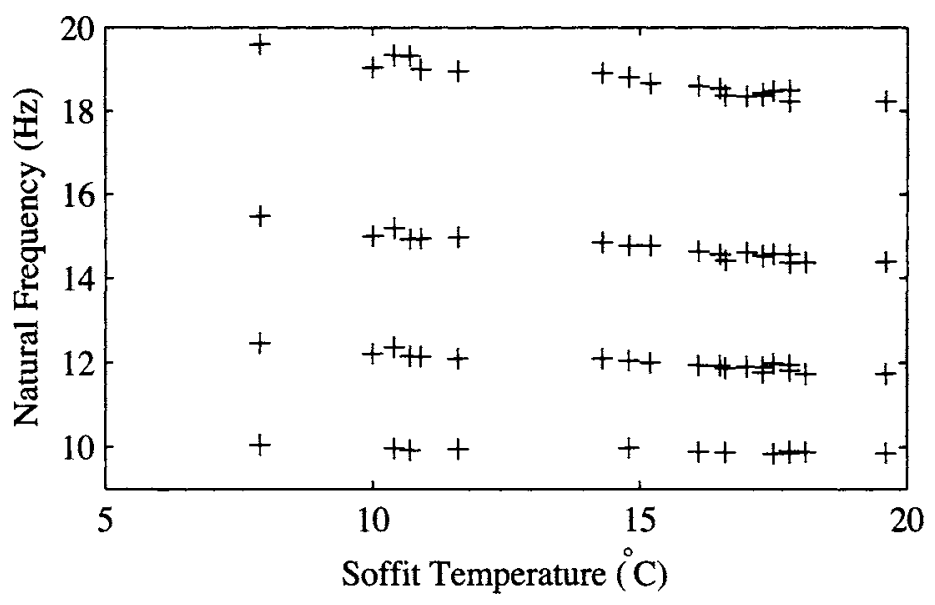

Figure 1. Variation of the 4 lowest natural frequencies of the New Haymills Bridge, Birmingham, UK.

The effect of frequency range The range of frequencies employed in damage location has a great influence on the resolution of the results and also the physical range of application. The great advantage in using low frequency vibration measurements is that the low frequency modes are generally global and so the vibration sensors may be mounted remotely from the damage site. Equally fewer sensors may be used. The problem with low frequency modes is that the spatial wavelengths of the modes are large, and typically are far larger than the extent of the damage. The spatial resolution of the damage identification scheme requires that there is a significant change in response between two adjacent potential damage sites. If low frequency modes are used then this resolution is closely related to the spatial wavelengths of the modes. Using high frequency excitation uses very local modes which are able to accurately locate damage, but only very close to the sensor and actuator position. Estimating accurate models at these high frequency ranges is also very difficult, and often changes in the response are used for damage identification. For example, Park et al. $(2000,2001)$ used changes in measured impedance to identify damage in civil structures and pipeline systems. Schulz et al. (1999) used high frequency transmissability to detect delaminations in composite structures.

Moving to even higher frequencies can also yield good results. Acoustic emission (Rogers, 2001) is a transient elastic wave, typically in the region of 50 to $500 \mathrm{kHz}$, and is able, for example, to detect the energy released when cracks propagate. One approach to damage assessment is to use physical models to deduce quantitative relationships between measured acoustic emission signals and the damage mechanism that cause them. Significant research has been undertaken to obtain a physical understanding of various source mechanisms (Scruby and Buttle, 1991) and the radiation pattern of bulk shear and longitudinal acoustic waves that they produce (Ono, 1991). The difficulty in using these models in inverse estimation procedures is the accuracy of these high frequency 
models, and the huge computational requirements. In recent years a pattern recognition philosophy has dominated, that relies on using large databases of empirical data from which correlations between measured acoustic emission signals and damage mechanisms are inferred. Many advanced signal processing algorithms have been employed to interpret experimental data. Damage location is often determined using time of flight methods, that require the events to be well separated in time, the wave speed to be approximately equal in all parts of the structure, and the effects of reflection and refraction to be insignificant. Examples of the use of acoustic emission for health monitoring are given by Rogers (2001), Atherton et al. (2004) and (Holford et al., 2001).

Damage magnitude A frequent problem that arises in model-based vibration-based damage detection, whether parametric or non-parametric, is the need for a very accurate mathematical model, so that it correctly captures the actual structural dynamic behaviour in some predetermined frequency range. Often in structural health monitoring the changes in the measured quantities caused by structural damage are smaller than those observed between the healthy (i.e. undamaged) structure and the mathematical model. Consequently, it becomes almost impossible to discern between inadequate modelling and actual changes due to damage. There are two alternative approaches to this problem. The first is to update the healthy model so that the correlation between the model and the measured data is improved. This approach requires that the errors that remain after updating are smaller in magnitude than the changes due to the damage. Furthermore the changes to the model should be physically meaningful, so that the updating process corrects actual model errors, and doesn't merely reproduce the measured data. The second approach is based on the use of (relative) differences between data measured on healthy and potentially damaged structure. In this case, assuming that the only changes in the structure are due to damage, the problem may be reduced to finding those parameters that reproduce the measured changes.

Non-linearity Many forms of damage cause a change in the stiffness non-linearity that qualitatively and quantitatively affects the dynamic response of a structure. For example, Nichols et al. (2003a,b) used the features of the chaotic response of a structure to detect changes in a joint. Adams and Nataraju (2002) gave a variety of features based on the non-linear dynamic response. Kerschen et al. (2003) considered model based estimation methods and identified the form of nonlinearity that is most likely present in the measured data. Meyer and Link (2003) identified a parametric non-linear model using harmonic balance and a model updating approach. A breathing crack, which opens and closes, can produce interesting and complicated non-linear dynamics. Brandon (1998) and Kisa and Brandon (2000) gave an overview of some of the techniques that may be applied. Many techniques to analyse the resulting non-linear dynamics are based on approximating the bilinear stiffness when the crack opens and closes. Linear approaches to damage estimation approximates a local reduction in the stiffness matrix of the beam. Since the non-linearity introduced by a crack is often weak, many of the common testing techniques will tend to linearise the response (Friswell and Penny, 2002). Sinusoidal forcing will tend to emphasise the non-linearity, and damage detection 
methods based on detecting harmonics of the forcing frequency have been proposed (Shen, 1998). In rotor dynamic applications these approaches are useful because the forcing is inherently sinusoidal (Dimarogonas, 1996). However in structural health monitoring applications this approach requires considerable hardware and software to implement, and also requires a lengthy experiment. Johnson et al. (2004) used a transmissibility approach that was insensitive to boundary condition non-linearities. Neild et al. (2003) investigated the potential of a time frequency analysis procedure to identify damage in concrete beams.

Although using the non-linear response has a huge potential in health monitoring, model based inverse approaches have a number of difficulties because of the high number of degrees of freedom required, and therefore the computational burden imposed. In practice, any realistic multi degree of freedom non-linear analysis would have to be based on a reduced order model of the structure. Furthermore, many of the difficulties outlined in this section for linear systems, are also a problem for non-linear systems.

Strength vs. stiffness The philosophy of damage detection using measured vibration data is based on the premise that the damage will change the stiffness of the structure. In some instances there is a significant difference between strength and stiffness. Indeed, estimating the remaining useful life of a component based on conclusions from a dynamic analysis is very difficult. For example, a concrete highway bridge will have steel reinforcement cables running in channels in the concrete. The cables are tensioned, either before or after the concrete has set, to ensure that the concrete remains in compression. One major failure mechanism is by the corrosion of these cables. Once the cables have failed the concrete has no strength in tension and so the bridge is liable to collapse. Unfortunately the stiffness of the bridge is mainly due to the concrete, and so the progressive corrosion of the cables is very difficult to identify from stiffness changes. Essentially the dynamics of the bridge do not change until it collapses.

\subsection{The Role of Simulation and Physical Testing}

Many of the algorithms suggested for damage location are tested on simulated data. It is necessary to fully test any method on both simulated and real data. The simulated tests are able to fully exercise the location methods, with the benefit that the answer is known. In simulation, far more damage cases may be used and the effect of errors may be fully investigated. The need for real testing arises because experimental work always produces errors and problems that are unexpected. For simulation to be useful, the errors that might be expected in real structures must be simulated. Thus, adding random noise to a model of the structure and then using the same model to identify the damage in not enough! Most identification schemes are able to cope very well with random noise, and although such simulations are important parts of the overall performance assessment of an algorithm, they are not sufficient. It is vital that systematic type errors are included in the simulation. Thus, discretisation errors may be included by generating the simulated measurements using a fine finite element model; the damage mechanism introduced to generate the measurements may be different to those modelled for the identification; or boundary conditions on the structure could be changed between the measured data set 
and the identification.

\subsection{A Simple Cantilever Beam Example}

A simulated cantilever beam example will be used to demonstrate some of the problems. Although the example is somewhat artificial it will highlight how easily methods fail even on very simple structures. Any practical method would have to be robust and should therefore succeed on simple structures, even though some systematic errors are included. This example also demonstrates the use of simulation in damage identification. Not all of the methods are tried and this example is not supposed to represent an extensive scientific evaluation of the methods. Its purpose is for illustration.

The beam has a cross section of $25 \mathrm{~mm} \times 50 \mathrm{~mm}$, a length of $1 \mathrm{~m}$ and is assumed to be rigidly clamped at one end. Only motion in the plane of the thinner beam dimension is considered. The beam has a Young's modulus of $210 \mathrm{GN} / \mathrm{m}^{2}$ and a mass density of $7800 \mathrm{~kg} / \mathrm{m}^{3}$.

The first test of any method is its application to a simulated example with no noise or systematic errors. Any parameter changes in the model should be identified exactly. The simulated measurements are assumed to be the relative changes in the lower natural frequencies of the beam and are taken from a model with 20 elements. The undamaged natural frequencies are taken from the uniform beam, whilst the damaged frequencies are derived from a model where the stiffness of element 4 has been reduced by $30 \%$. Table 1 gives the damaged and undamaged natural frequencies, showing that the $30 \%$ damage only results in a $2.4 \%$ change in natural frequency at most. These small frequency changes are typical in damage location and are one of the major difficulties in the identification of the location of damage. Measurement noise, environmental factors and structure nonstationarity can easily lead to incorrect conclusions on damage location.

Table 1. Natural frequencies of the simulated undamaged and damaged beam.

\begin{tabular}{|c|c|c|c|}
\hline Mode No. & Undamaged $(\mathrm{Hz})$ & Damaged $(\mathrm{Hz})$ & Difference(\%) \\
\hline 1 & 20.96 & 20.45 & 2.39 \\
2 & 131.3 & 131.1 & 0.15 \\
3 & 367.7 & 366.6 & 0.31 \\
4 & 720.6 & 711.3 & 1.29 \\
5 & 1191 & 1172 & 1.61 \\
6 & 1780 & 1762 & 1.02 \\
7 & 2487 & 2479 & 0.32 \\
8 & 3313 & 3303 & 0.30 \\
\hline
\end{tabular}

The standard sensitivity approach based on modal data will now be used to identify the damage. The set of candidate parameters is chosen to be relatively large and consists of the stiffness of each of the 20 elements. If the relative changes to the first 8 natural frequencies are used as the measurements then the identification of the parameters is underdetermined. In this case some form of regularisation must be employed. Figure 2 shows the change in element stiffness required to reproduce the damaged natural frequencies, using a minimum norm constraint on the parameter changes. Although the largest 
stiffness change occurs at element 4 the identified damage is spread over the whole beam, and there are some significant increases in stiffness. Note that this is the ideal case with no measurement noise or modelling errors. Suppose that, by some means, the damage is known to be somewhere in the eight elements closest to the fixed end. The number of parameters is now reduced to eight, the same as the number of natural frequencies. There is now a unique solution to the estimation problem and this solution is given in Figure 3. Note that the stiffness of elements 9 to 20 cannot change, but are included in Figure 3 for easy comparison. The damage has clearly been correctly located to element 4. However the magnitude of the damage is incorrect because the estimation is based on the sensitivity matrix which is a linear approximation to the residual. The other seven parameters are non-zero for the same reason.

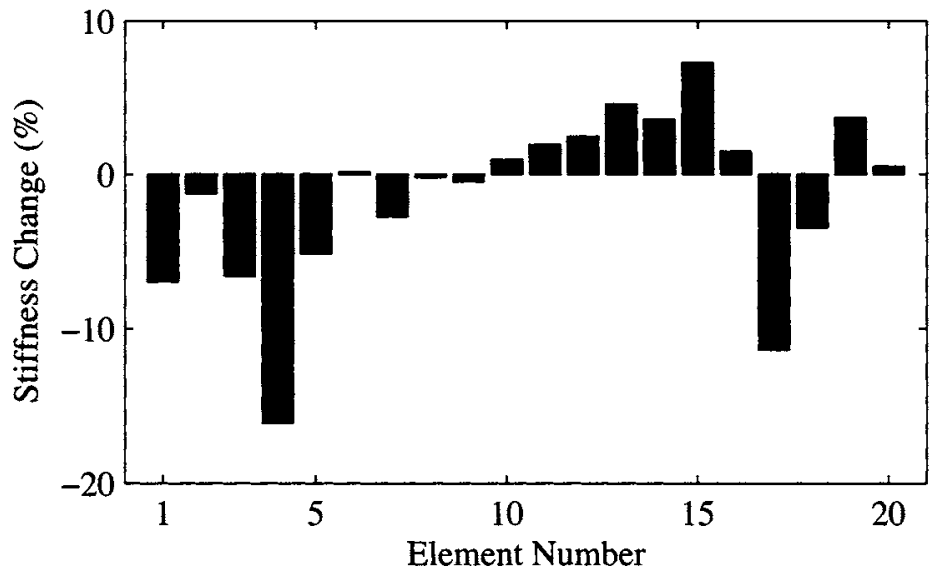

Figure 2. The change in element stiffness estimated for the cantilever beam example with no noise and a minimum norm constraint.

\section{Regularisation}

The advantages of sensitivity type model updating methods have been highlighted in this chapter. However there are significant differences in the application of these methods in model updating and damage location, which necessitates different methods of regularisation. In both cases the number of potential parameters is very large and the estimation process is likely to be ill-conditioned unless the physical understanding can be used to introduce extra information.

In model updating, the number of parameters may be reduced by only including those parameters that are likely to be in error. Thus if a frame structure is updated, the beams are likely to be modelled accurately but the joints are more difficult to model. It would therefore be sensible to concentrate the uncertain parameters to those associated with the joints. Even so, a large number of potential parameters may be generated, the measurements may still be reproduced and the parameters are unlikely to be identified 


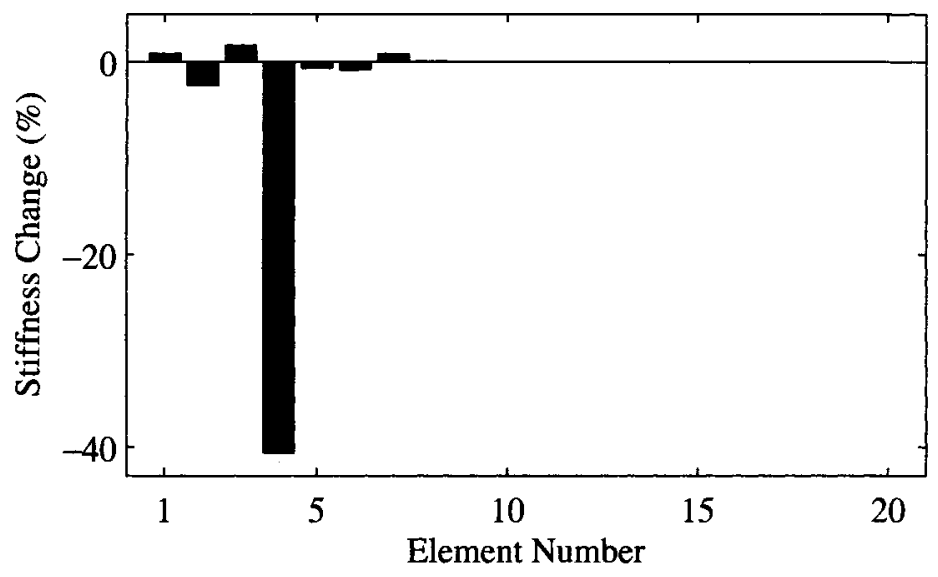

Figure 3. The change in element stiffness estimated for the cantilever beam example with no noise, but only 8 non-zero parameters.

uniquely. In this situation all the parameters are changed, and regularisation must be applied to generate a unique solution (Friswell et al., 2001). Regularisation generally applies extra constraints to the parameter estimation problem to ensure a unique solution. Applying the standard Moore-Penrose pseudo inverse is a type of regularisation where the parameter vector with the minimum norm is chosen. The parameter changes may be weighted separately to give a weighted least squares problem, where the penalty function is a weighted sum of squares of the measurement errors and the parameter changes. Such weighting may also be extended to include minimising the difference between equivalent parameters that are nominally equal in different substructures such as joints. Although using parametric models can reduce the number of parameters considerably, for damage location there will still be a large number of parameters. Most regularisation techniques rely on minimum norm type solutions that will tend to spread the identified damage over a large number of parameters. Using subset selection, where only the optimum subset of the parameters are used for the estimation (Friswell et al., 1997), has been used for model updating and also for damage location.

\subsection{Tikhonov Regularisation}

The treatment of ill-conditioned, noisy systems of equations is a problem central to finite element model updating (Ahmadian et al., 1998). Such equations often arise in the correction of finite element models by using vibration measurements. The regularisation problem centres around the linear equation,

$$
\mathbf{A x}=\mathbf{b}
$$

where $\mathbf{x}$ is a vector of the $m$ parameter changes we wish to determine, and $\mathbf{b}$ is a vector of $n$ residual quantities derived from the measured data and the current estimate of the model. Note that the iteration index $j$ has been dropped from the expressions 
in Section 1.2 , and $\delta \theta$ has been replaced by $\mathbf{x}$. In model updating the relationship between the measured output (for example, natural frequencies, mode shapes, or the frequency response function) is generally non-linear. In this case the problem is linearised using a Taylor series expansion and iteration performed until convergence. When $\mathbf{b}$ is contaminated with additive, independent random noise with zero mean, it is well known that the least-squares solution, $\mathbf{x}_{L S}$, is unique and unbiased provided that $\operatorname{rank}(\mathbf{A})=m$. When $\mathbf{A}$ is close to being rank deficient then small levels of noise may lead to a large deviation in the estimated parameters from its exact value. The solution is said to be unstable and Equation (2.1) is ill-conditioned

A different problem occurs when $m>n$ so that Equation (2.1) is under-determined and there are an infinite number of solutions. The Moore-Penrose pseudo-inverse in the form,

$$
\mathbf{x}_{L S}=\mathbf{A}^{\top}\left[\mathbf{A} \mathbf{A}^{\top}\right]^{-1} \mathbf{b}
$$

provides the solution of minimum norm, as does singular value decomposition (SVD). For the case when $\operatorname{rank}(\mathbf{A})=r<\min (m, n)$, the SVD will again result in the minimum norm solution. This is a form of regularisation which has been widely applied in the model updating community. Unfortunately minimum norm solutions rarely lead to physically meaningful updated parameters.

Side Constraints Model updating often leads to an ill-conditioned parameter estimation problem, and an effective form of regularisation is to place constraints on the parameters. This could be that the deviation between the parameters of the updated and the initial model are minimised, or differences between parameters could be minimised. For example, in a frame structure a number of ' $\mathrm{T}$ ' joints may exist that are nominally identical. Due to manufacturing tolerances the parameters of these joints will be slightly different, although these differences should be small. Therefore a side constraint is placed on the parameters, so that both the residual and the differences between nominally identical parameters are minimised. Thus if Equation (2.1) generates the residual, the parameter is sought which minimises the quadratic cost function,

$$
J(\mathbf{x})=\|\mathbf{A} \mathbf{x}-\mathbf{b}\|^{2}+\lambda^{2}\|\mathbf{C} \mathbf{x}-\mathbf{d}\|^{2}
$$

for some matrix $\mathbf{C}$, vector $\mathbf{d}$ and regularisation parameter $\lambda$. The regularisation parameter is chosen to give a suitable balance between the residual and the side constraint. For example, if there were only two parameters, which were nominally equal, then

$$
\mathbf{C}=[1-1]
$$

Minimising Equation (2.3) is equivalent to minimising the residual of

$$
\left[\begin{array}{c}
\mathbf{A} \\
\lambda \mathbf{C}
\end{array}\right] \mathbf{x}=\left\{\begin{array}{c}
\mathbf{b} \\
\lambda \mathbf{d}
\end{array}\right\}
$$

Equation (2.5) then replaces Equation (2.1), although with the significant difference that Equation (2.5) is generally over-determined, whereas Equation (2.1) if often underdetermined. The constraints should be chosen to satisfy Morozov's complementation 
condition

$$
\operatorname{rank}\left[\begin{array}{c}
\mathbf{A} \\
\mathbf{C}
\end{array}\right]=m
$$

which ensures that the coefficient matrix in Equation (2.5) is full rank.

The Singular Value Decomposition The singular value decomposition (SVD) of $\mathbf{A}$ may be written in the form,

$$
\mathbf{A}=\mathbf{U} \boldsymbol{\Sigma} \mathbf{V}^{\top}=\sum_{i=1}^{m} \sigma_{i} \mathbf{u}_{i} \mathbf{v}_{i}^{\top}
$$

where $\mathbf{U}=\left[\mathbf{u}_{1} \mathbf{u}_{2} \ldots \mathbf{u}_{n}\right]$ and $\mathbf{V}=\left[\mathbf{v}_{1} \mathbf{v}_{2} \ldots \mathbf{v}_{m}\right]$ are $n \times n$ and $m \times m$ orthogonal matrices and

$$
\boldsymbol{\Sigma}=\operatorname{diag}\left(\sigma_{1}, \sigma_{2}, \ldots, \sigma_{m}\right)
$$

where the singular values, $\sigma_{i}$, are arranged in descending order $\left(\sigma_{1} \geq \sigma_{2} \geq \ldots \geq \sigma_{m}\right)$. In ill-posed problems two commonly occurring characteristics of the singular values have been observed; the singular values decay steadily to zero with no particular gap in the spectrum, and the left and right singular vectors $\mathbf{u}_{i}$ and $\mathbf{v}_{i}$ tend to have more sign changes in their elements as the index $i$ increases.

The solution for the parameters using the SVD is

$$
\mathbf{x}=\sum_{i=1}^{m} \frac{\mathbf{u}_{i}^{\top} \mathbf{b}}{\sigma_{i}} \mathbf{v}_{i}
$$

Thus the components of $\mathbf{A}$ corresponding to the low singular values have only a small contribution to $\mathbf{A}$ but a large contribution to the estimated parameters. The elements of these singular vectors (corresponding to the low singular values) are also generally highly oscillatory. Equation (2.9) shows the noise will be amplified when $\sigma_{i}<\mathbf{u}_{i}^{\top} \mathbf{b}$, and this may be used to decide where to truncate the singular values. If $\mathbf{A}$ does not contain noise then the singular values will decay to zero whereas the $\mathbf{u}_{i}^{\top} \mathbf{b}$ terms will decay to the noise level. Ahmadian et al. (1998); Hemez and Farhat (1995) consider this approach in more detail.

The standard SVD is incapable of taking account of the side constraint, as this requires the generalised SVD. Space does not permit a full explanation of the generalised SVD, and the reader is referred to Hansen (1994) for more complete detail of the decomposition. In Equation (2.5), $\mathbf{A}$ and $\mathbf{C}$ are decomposed as

$$
\mathbf{A}=\mathbf{U}\left[\begin{array}{ll}
\mathbf{I} & \mathbf{0} \\
\mathbf{0} & \boldsymbol{\Sigma}
\end{array}\right] \mathbf{X}^{-1} \quad \mathbf{C}=\mathbf{V}[\mathbf{0} \mathbf{M}] \mathbf{X}^{-1}
$$

where $\mathbf{X}$ is a non-singular $m \times m$ matrix. $\mathbf{U}$ and $\mathbf{V}$ are $n \times m$ and $p \times p$ respectively, and their columns are orthogonal (but they are not related to the matrices $\mathbf{U}$ and $\mathbf{V}$ of the standard SVD) and $n \geq m \geq p$. The matrices $\boldsymbol{\Sigma}$ and $\mathbf{M}$ are

$$
\boldsymbol{\Sigma}=\operatorname{diag}\left(\sigma_{1}, \sigma_{2}, \ldots, \sigma_{p}\right) \quad \mathbf{M}=\operatorname{diag}\left(\mu_{1}, \mu_{2}, \ldots, \mu_{p}\right)
$$


where $1 \geq \sigma_{1} \geq \sigma_{2} \geq \ldots \geq \sigma_{p} \geq 0$ and $0 \leq \mu_{1} \leq \mu_{2} \leq \ldots \leq \mu_{p} \leq 1$, and $\sigma_{i}$ and $\mu_{i}$ are normalised so that,

$$
\sigma_{i}^{2}+\mu_{i}^{2}=1
$$

The $p$ generalised singular values of $\left[\begin{array}{l}\mathbf{A} \\ \mathbf{C}\end{array}\right]$, in decreasing order, are then

$$
\gamma_{i}=\frac{\sigma_{i}}{\mu_{i}} .
$$

The solution to Equation (2.5) is then

$$
\mathbf{x}=\sum_{i=1}^{p} \frac{\gamma_{i}^{2}}{\gamma_{i}^{2}+\lambda^{2}} \frac{\mathbf{u}_{i}^{\top} \mathbf{b}}{\sigma_{i}} \mathbf{v}_{i}+\sum_{i=p+1}^{m}\left(\mathbf{u}_{i}^{\top} \mathbf{b}\right) \mathbf{v}_{i}
$$

The regularisation parameter, $\lambda$, has the effect of damping the effect of the lower singular values (lower than about $\lambda$ ) and thus smoothing the solution. The expansion in terms of the SVD, (2.14), may also be used to specify a solution as a truncated SVD. If, instead of specifying $\lambda$, the series is truncated by only keeping the largest $k$ singular values, then the solution is

$$
\mathbf{x}=\sum_{i=1}^{k} \frac{\mathbf{u}_{i}^{\top} \mathbf{b}}{\sigma_{i}} \mathbf{v}_{i}+\sum_{i=p+1}^{m}\left(\mathbf{u}_{i}^{\top} \mathbf{b}\right) \mathbf{v}_{i}
$$

Picard's condition may be used to choose $k$, and the expansion is truncated when $\frac{\mathbf{u}_{i}^{\top} \mathbf{b}}{\sigma_{i}}$ becomes large.

' $\mathbf{L}$ ' Curves One way of obtaining the optimum value of the regularisation parameter in the presence of correlated noise is to define an upper bound for the side constraint and minimise the residue,

$$
\min _{\mathbf{x}}\|\mathbf{A} \mathbf{x}-\mathbf{b}\| \quad \text { subject to } \quad\|\mathbf{C} \mathbf{x}-\mathbf{d}\| \leq \gamma,
$$

or alternatively to set a limit for the residue and minimise the deviation from the side constraint,

$$
\min _{\mathbf{x}}\|\mathbf{C x}-\mathbf{d}\| \quad \text { subject to }\|\mathbf{A x}-\mathbf{b}\| \leq \varepsilon .
$$

Of course the success of this approach is highly dependent on the physical insight of the analyst in determining the allowable constraint violation or measurement error (residue magnitude).

A different approach is to plot the norm of the side constraint, $\|\mathbf{C x}-\mathbf{d}\|$, against the norm of the residue, $\|\mathbf{A x}-\mathbf{b}\|$, obtained by minimising the penalty function Equation (2.3) for different values of $\lambda$. Hansen (1992) showed that the norm of the side constraint is a monotonically decreasing function of the norm of the residue, and any point $(\varepsilon, \gamma)$ on the curve is a solution to the two constrained least-squares problems 
Equations (2.16) and (2.17). He pointed out that for a reasonable signal-to-noise ratio and the satisfaction of the Picard condition, the curve is approximately vertical for $\lambda<\lambda_{\text {opt }}$, and soon becomes a horizontal line when $\lambda>\lambda_{\text {opt, }}$, with a corner near the optimal regularisation parameter $\lambda_{\text {opt }}$. The curve is called the 'L'-curve because of this behaviour. The optimum value of the regularisation parameter, $\lambda_{\text {opt }}$, corresponds to the point with maximum curvature at the corner of the log-log plot of the 'L'-curve. This point represents a balance between confidence in the measurements and the analyst's intuition.

Cross-Validation The idea of cross-validation is to maximise the predictability of the model by choice of the regularisation parameter $\lambda$. A predictability test can be arranged by omitting one data point, $b_{k}$, at a time and determining the best parameter estimate using the other data points, by minimising Equation (2.3). Then for each of the estimates, predict the missing data and find the value of $\lambda$ that on average predicts the $b_{k}$ best, in the sense of minimising the cross-validation function

$$
V_{0}(\lambda)=\frac{1}{n} \sum_{k=1}^{n}\left(b_{k}-\tilde{b}_{k}(\lambda)\right)^{2}
$$

where $\tilde{b}_{k}(\lambda)$ is the estimate of $b_{k}$ obtained from the remaining data. This is the method of cross-validation. Equation (2.18) is equivalent to (Ahmadian et al., 1998),

$$
V_{0}(\lambda)=\frac{1}{n}\left\|[\operatorname{diag}(\mathbf{I}-\mathbf{R}(\lambda))]^{-1}[\mathbf{A x}(\lambda)-\mathbf{b}]\right\|^{2}
$$

where

$$
\mathbf{R}(\lambda)=\mathbf{A}\left[\mathbf{A}^{\top} \mathbf{A}+\lambda^{2} \mathbf{C}^{\top} \mathbf{C}\right]^{-1} \mathbf{A}^{\top}
$$

and diag denotes the matrix with zeros assigned to the off-diagonal terms.

\subsection{Subset Selection}

One solution to the problem of ill-conditioning is to select only a subset of the parameters for updating (Friswell et al., 1997). The parameters that are chosen are those to which the response data is sensitive, but the parameters must also be able to correct the errors in the model. Parameter subset selection is a technique that selects the best subset of parameters from a candidate set, utilising some application dependent cost function that provides a measure of goodness of each subset. Often, these techniques only obtain a sub-optimal estimate of the best subsets in some sense due to the excessive computational burden posed by the original problem. These techniques are firmly rooted in statistics and related fields (Millar, 1990), although recently applications in structural mechanics have appeared. Friswell et al. (1997) gave an overview of subset selection and also proposed the use of this technique for damage detection. They suggested an approach based on forward parameter subset selection, which is especially suited to local damage, and applied the method to a simulated cantilever beam example with physical parameters corresponding to either element or node properties. Different selection and iteration strategies were evaluated, and the case where multiple measurement sets are 
available was handled by computing the principal angles between two vector subspaces. Fritzen et al. (1998) used a orthogonalisation scheme for subset selection.

In damage location statistical methods and performance measures have been used that work on a similar principle (Cawley and Adams, 1979; Cawley et al., 1978; Friswell et al., 1994). Only a limited number of sites are assumed to be damaged, and the model updated based on the reduced number of parameters. This process is repeated for all possible combinations of damage site, and possibly even damage mechanism. The results from all the updated models are compared and the one that best matches the measured data is chosen.

The major problem with both subset selection and the statistical type approach, is that many smaller model updating exercises have to be performed. To optimally derive the best set of parameters, or the best damage location, requires the evaluation of many subsets of parameters. With a large number of parameters evaluating all subsets of even 2 or 3 parameters can become daunting. Thus sub-optimal methods must be used to derive good, but not necessarily the best, subsets of the parameters. In the forward approach parameters are chosen one at a time, and the parameters selected previously are retained. However there is no guarantee that the optimal subset will be found. The number of candidate damage locations may be controlled based on the expected reduction in the residual (Millar, 1990). The addition of a parameter to a previously selected subset inevitably reduces the residual terms, and thus there is a trade off between the number of parameters selected and the magnitude of the residual. Often only a single damage location will be required, in which case the optimal parameter may be determined. Often a reasonable number of parameter subsets (say between 3 and 20) are selected for more detailed study (Millar, 1990). Friswell et al. (1997) reviewed the relationship between subset selection and matrix decomposition, and also expanded the methods to parameter groups using subspace angles. Titurus et al. (2003b) considered the weighting requirements within the inner product defining the subspace angles, following the work of Knyazev and Argentati (2002).

The process of subset selection will now be described. It should be highlighted that the standard approach to subset selection is not iterative, but only uses Equation (2.1), evaluated at the initial parameter values. It would be possible to update each candidate parameter set until convergence, and then compare the performance of the different subsets, although it practice the computational cost is prohibitive. As the model parameters are usually local in nature and may also allow for different damage mechanisms, parameter subset selection selects parameters from $\mathbf{x}$ that identify both the damage location and mechanism. This formulation requires the selection of the optimum parameter subset from $\mathbf{x}$. The most straightforward approach is to use an exhaustive search where all $\left(2^{m}-1\right)$ possible cases have to be searched. The number of cases renders this approach computational intensive and thus impractical in many real situations. Consequently sub-optimal schemes have to be used. An additional problem is that the addition of a parameter to a previously selected subset inevitably reduces the residual generated by Equation (2.1). Thus there is a trade off between the number of parameters selected and the magnitude of the residual. 
Equation (2.1) may be written as

$$
\mathbf{A x}=\left[\mathbf{a}_{1}, \mathbf{a}_{2}, \ldots, \mathbf{a}_{m}\right] \mathbf{x}=\mathbf{b}
$$

The case of a single damage location leads to a simplified version of above philosophy. When only one parameter is selected, the optimum parameter is that which best fits the changes due to damage characterised by the vector $\mathbf{b}$ in Equation (2.21). Thus, the goal is to find the column $\mathbf{a}_{j}$ of matrix $\mathbf{A}$ that minimises

$$
J=\left\|\mathbf{b}-\mathbf{a}_{j} \hat{x}_{j}\right\|^{2}
$$

where $\hat{x}_{j}$ is the least squares estimate of the $j$ th parameter in $\mathbf{x}$. Friswell et al. (1997) showed that minimising Equation (2.22) is equivalent to finding the column of $\mathbf{A}$ that minimises the angle with $\mathbf{b}$. Hence the best parameter is the $j$ th and found by

$$
\min \left(\left\{\psi_{1}, \psi_{2}, \ldots, \psi_{m}\right\}\right) \Rightarrow \hat{x}_{j}, \mathbf{a}_{j}
$$

where

$$
\cos ^{2} \psi_{i}=\frac{\left(\mathbf{a}_{i}^{\top} \mathbf{b}\right)^{2}}{\left(\mathbf{a}_{i}^{\top} \mathbf{a}_{i}\right)\left(\mathbf{b}^{\top} \mathbf{b}\right)}, \quad i=1,2, \ldots, m
$$

and $\psi_{i}$ is the angle between vectors $\mathbf{a}_{i}$ and $\mathbf{b}$. This step is part of a general technique used in damage detection (Friswell et al., 1997) and is called forward parameter subset selection. This is a sub-optimal technique of subset selection, starting with the above step and continuing by additional parameter searches where the already selected parameters are retained. For subsequent steps a new modified problem is created, respecting the previous parameter selections. Suppose the single parameter with index $j_{1}$ has been chosen, then the parameter estimate $\hat{x}_{j_{1}}$ and residual $\varepsilon$ are

$$
\hat{x}_{j_{1}}=\frac{\mathbf{a}_{j_{1}}^{\top} \mathbf{b}}{\mathbf{a}_{j_{1}}^{\top} \mathbf{a}_{j_{1}}} \Rightarrow \varepsilon=\mathbf{b}-\hat{x}_{j_{1}} \mathbf{a}_{j_{1}} .
$$

Note that $\varepsilon$ is orthogonal to $\mathbf{a}_{j_{1}}$. A new parameter is then sought by considering the subspace defined by columns of $\mathbf{A}$, but orthogonal to $\mathbf{a}_{j_{1}}$. The modified problem is defined as (Friswell et al., 1997),

$$
\mathbf{a}_{j} \rightarrow \mathbf{a}_{j}-\alpha_{j} \mathbf{a}_{j_{1}}, \quad \mathbf{b} \rightarrow \mathbf{b}-\hat{x}_{j_{1}} \mathbf{a}_{j_{1}}
$$

where

$$
\alpha_{j}=\left(\mathbf{a}_{j_{1}}^{\top} \mathbf{a}_{j}\right) /\left(\mathbf{a}_{j_{1}}^{\top} \mathbf{a}_{j_{1}}\right)
$$

A second parameter may now be selected by means of the modified problem defined by Equation (2.26), where $j \neq j_{1}$. Further parameters may be selected in the same way. An algorithm is thus created to search for the best parameter subset, denoted by $\left[\hat{x}_{j_{1}}, \hat{x}_{j_{2}}, \ldots, \hat{x}_{j_{p}}\right]$, that minimises the cost function,

$$
J_{p}=\left\|\mathbf{b}-\sum_{i=1}^{p} \hat{x}_{j_{i}} \mathbf{a}_{j_{i}}\right\|^{2}
$$


This cost function is also employed in Efroymson's algorithm for forward subset selection, which focuses on adding or removing parameter selections from chosen subsets. Thus the number of candidate damage locations may be controlled based on the expected reduction in the residual (Millar, 1990; Friswell et al., 1997). Since only single damage location cases will be examined in detail here this subject will not be considered further.

Weighting The final theoretical aspect is the need for weighting when Equation (1.5) is used for damage location, and two types of weighting will be considered. First, weighting is needed to handle the different numerical values corresponding to the different modal quantities. Thus, only relative, or percentage changes in the modal quantities, due to damage, will be employed. The second type of weighting arises as a result of combining two different entities in the sensitivity matrix, namely natural frequencies and mode shapes for complete damage location. The experimental origin of the measurements means that the errors in mode shape estimation are usually greater than the errors in natural frequency estimation. This weighting is employed in the calculation of the subspace angles between the vector $\delta \mathbf{z}$ and the columns of the matrix $\mathbf{A}$, Equation (2.23), and is based on the weighted scalar product (Knyazev and Argentati, 2002). The procedure is also called the scalar A-based product and has its origins in statistics (note that the $A$ in the name of this product has nothing to do with the matrix $\mathbf{A}$ in Equation (2.21)). Knyazev and Argentati (2002) studied this scalar product in the context of the numerically stable computation of principal angles between two linear subspaces. The scalar A-based inner product is defined as

$$
(\mathbf{x}, \mathbf{y})_{A}=\left(\mathbf{x}, \mathbf{A}_{W} \mathbf{y}\right)=\mathbf{y}^{\top} \mathbf{A}_{W} \mathbf{x}
$$

where $\mathbf{x}, \mathbf{y} \in \Re^{n}$ are vectors, and $\mathbf{A}_{W} \in \Re^{n \times n}$ is a symmetric, positive definite matrix. A-based vector and matrix norms, $\|. .\|_{A}$, may be defined as

$$
\|\mathbf{x}\|_{A}=\sqrt{(\mathbf{x}, \mathbf{x})_{A}}=\left\|\mathbf{A}_{W}^{1 / 2} \mathbf{x}\right\|, \quad\|\mathbf{B}\|_{A}=\left\|\mathbf{A}_{W}^{1 / 2} \mathbf{B} \mathbf{A}_{W}^{-1 / 2}\right\|
$$

where $\mathbf{B} \in \Re^{n \times n}$ is an arbitrary matrix.

The applicability of this type of product for damage location based on the additional use of mode shape sensitivities in $\mathbf{S}$ and mode shape differences due to damage will be studied for an experimental, geometrically symmetric structure in a later section. Since $\delta \mathbf{z}$ is derived from experimental data, and assuming that the mass distribution does not change with damage, no additional scaling of individual mode shapes, with respect to other modes, will be employed. Since the natural frequencies are measured much more accurately than the mode shapes, the natural frequencies should be used to determine the candidate damage locations. The $\mathrm{A}$ weighting on the mode shapes is then used for geometrically symmetric structures to ensure that the most likely damage location from among the candidate locations identified from the natural frequencies is chosen. The weighting of the mode shapes is increased until a perceptible difference occurs between these candidate locations, but is kept as low as possible to reduce the effect of the noise on the mode shapes. 


\subsection{The Simple Cantilever Beam Example Revisited}

The simple cantilever beam example of Section 1.7 will be used to demonstrate some of the properties of the methods given in this section. Candidate parameters now include element mass, and discrete mass and springs, as well as the element stiffness. Figure 4 shows the angles between the columns of the sensitivity matrix of the initial finite element model and the vector of the relative changes in the first 8 natural frequencies due to the damage. Clearly the column relating to the stiffness of element 4 has a small angle, although it is not zero because the method is based on a first order approximation and the extent of the damage (30\%) is large. Changing the mass of element 17 is also able to model the measured changes accurately. This is a problem that relates to the symmetry of the beam, and the fact than no spatial information is incorporated into the measurements. Mode shapes could also be incorporated into the measurement vector, although the accuracy with which they could be measured may be insufficient to show a change in mode shape due to damage. This is an example of the more general problem, where damage or changes of parameters at more than one location causes the same changes in the lower natural frequencies.

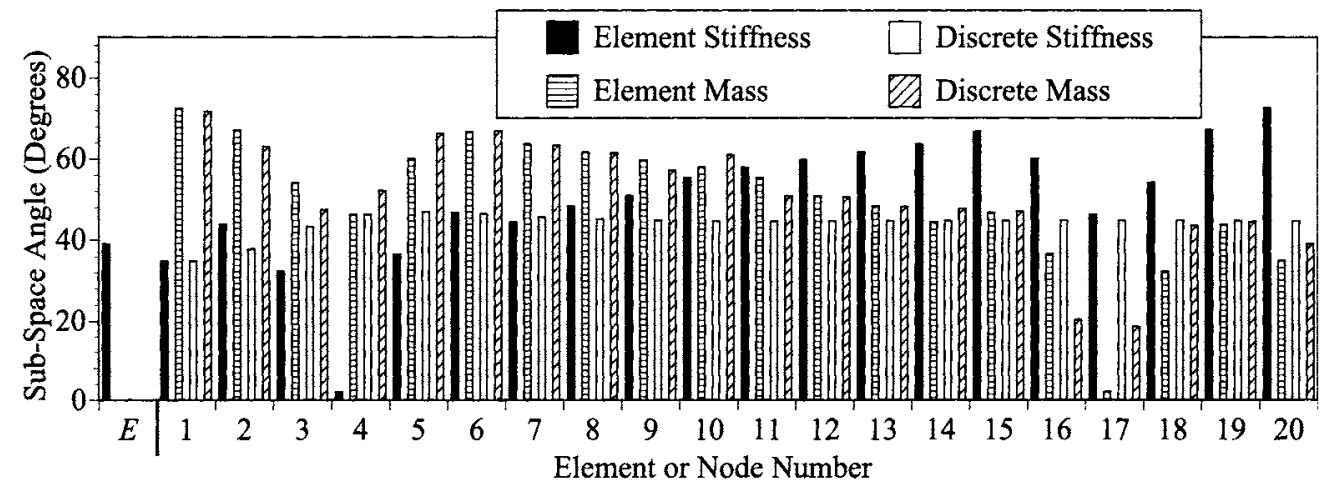

Figure 4. Subspace angles for a $30 \%$ change to the stiffness of element 4.

Subset selection is next demonstrated on an example where damage is introduced at 2 locations. A $0.1 \mathrm{~kg}$ mass is added to node 12 , in addition to the $30 \%$ stiffness change to element 4 . This example does not include any measurement noise or modelling errors. Table 2 shows the results when the best subsets of 1,2 and 3 parameters are chosen. The parameters are specified by type and element or node number. Thus $(\rho A)_{17}$ is the mass / unit length of element $17,(E I)_{4}$ is the stiffness of element $4, k_{1}$ is a discrete spring at node 1 and $m_{12}$ is a discrete mass at node 12 . At each stage the 2 best parameters are chosen. The residuals under the first 2 parameters relate to the values when a subset of size 1 or 2 is selected. Also shown are the residuals after convergence based on optimising the values of the chosen parameters. From the values of the residuals, it is clear that the two correct parameters should be selected. A number of other parameter subsets have small residuals and the addition of random noise would make the selection of the best subset more difficult. 
Table 2. The selection of three parameters for the beam example.

\begin{tabular}{|c|c|c|c|c|c|c|c|c|}
\hline \multicolumn{3}{|c|}{ Parameter 1} & \multicolumn{3}{|c|}{ Parameter 2} & \multicolumn{3}{|c|}{ Parameter 3} \\
\hline & $\begin{array}{l}\text { Res- } \\
\text { idual }\end{array}$ & $\begin{array}{l}\text { Conv- } \\
\text { erged }\end{array}$ & & $\begin{array}{l}\text { Res- } \\
\text { idual }\end{array}$ & $\begin{array}{l}\text { Conv- } \\
\text { erged }\end{array}$ & & $\begin{array}{l}\text { Res- } \\
\text { idual }\end{array}$ & $\begin{array}{l}\text { Conv- } \\
\text { erged }\end{array}$ \\
\hline$(\rho A)_{17}$ & 154.6 & 160.4 & $\begin{array}{c}m_{12} \\
m_{8}\end{array}$ & $\begin{array}{l}1.49 \\
8.70\end{array}$ & $\begin{array}{r}0.782 \\
4.76\end{array}$ & $\begin{array}{c}m_{8} \\
(\rho A)_{12} \\
m_{12} \\
(\rho A)_{12}\end{array}$ & $\begin{array}{l}1.21 \\
1.48 \\
1.21 \\
8.68\end{array}$ & $\begin{array}{c}0.701 \\
0.286 \\
0.701 \\
4.75\end{array}$ \\
\hline$(E I)_{4}$ & 154.7 & 160.5 & $\begin{array}{c}m_{12} \\
m_{8}\end{array}$ & 1.49 & 0.000 & $\begin{array}{c}m_{8} \\
(\rho A)_{12} \\
m_{12} \\
(\rho A)_{12}\end{array}$ & $\begin{array}{l}1.20 \\
1.48 \\
1.20 \\
8.69 \\
\end{array}$ & $\begin{array}{c}0.000 \\
0.000 \\
0.000 \\
5.35 \\
\end{array}$ \\
\hline
\end{tabular}

\section{Parameterisation of Models of Damage}

Damage usually causes a reduction in the local stiffness of the structures. One option is to model this a reduction in stiffness at the element or substructure level. This equivalent modelling approach is often sufficient for the identification of local damage using low frequency vibration measurements. This section considers more detailed models of damage that have parameters that may be identified using inverse methods.

\subsection{Crack Models}

The modelling of cracks in beam structures and rotating shafts has been a significant research topic. The models fall into three main categories; local stiffness reduction, discrete spring models, and complex models in two or three dimensions. Dimarogonas (1996); Ostachowicz and Krawczuk (2001) gave comprehensive surveys of crack modelling approaches. The simplest methods for finite element models reduce the stiffness locally, for example by reducing a complete element stiffness to simulate a small crack in that element (Mayes and Davies, 1984). This approach suffers from problems in matching damage severity to crack depth, and is affected by the mesh density. An improved method introduces local flexibility based on physically based stiffness reductions, where the crack position may be used as a parameter for identification purposes. The second class of methods divides a beam type structure into two parts that are pinned at the crack location and the crack is simulated by the addition of a rotational spring. These approaches are a gross simplification of the crack dynamics and do not involve the crack size and location directly. The alternative, using beam theory, is to model the dynamics close to the crack more accurately, for example producing a closed form solution giving the natural frequencies and mode shapes of cracked beam directly or using differential equations with compatible boundary conditions satisfying the crack conditions (Christides and Barr, 1984; Sinha et al., 2002; Lee and Chung, 2001). Friswell and Penny (2002) compared several of the simple cracks models that may be used for health monitoring, for both the linear and non-linear response. Alternatively two or three dimensional finite element meshes for beam type structures with a crack may be used. Meshless approaches 
may also be used, but are more suited to crack propagation studies. No element connectivity is required and so the task of remeshing as the crack grows is avoided, and a growing crack is modelled by extending the free surfaces corresponding to the crack (Belytschko et al., 1995). However the compuational cost of these meshless methods generally exceeds that of conventional finite element analysis (FEA). Rao and Rahman (2001) avoided this difficulty by coupling a meshless region near the crack with an FEA model in the remainder of the structure. The two and three dimensional approaches produce detailed and accurate models but are a complicated and computational intensive approach to model simple structures like beams, and are unlikely to lead to practical algorithms for damage identification.

Models of open cracks Although the geometry of a crack can be very complicated, the contention in this paper is that for low frequency vibration only an effective reduction in stiffness is required. Thus, for comparison, a simple model of an open crack, which is essentially a saw cut, will be used. This will allow the comparison of models using beam elements, with those using plate elements. Only a selection of beam models will be used, that illustrate the fact that many beam models are able to model the effect of the crack at low frequencies.

Two standard approaches using beam elements are shown in Figure 5. In the first approach, the stiffness of a single element is reduced, which requires a fine mesh, and also the derivation of the effect of a crack on the element stiffness. In the second approach, the beam is separated into two halves at the crack location. The beam sections are then pinned together and a rotational spring used to model the increased flexibility due to the crack. Translational springs may also be used in place of the pinned constraint. The major difficulties with this approach is that a finite element node must be place at the crack location, requiring remeshing for health monitoring applications, and the relationship between the spring stiffness and crack depth needs to be derived.

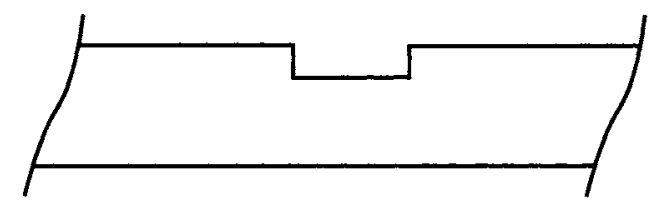

Reduction in Element Stiffness

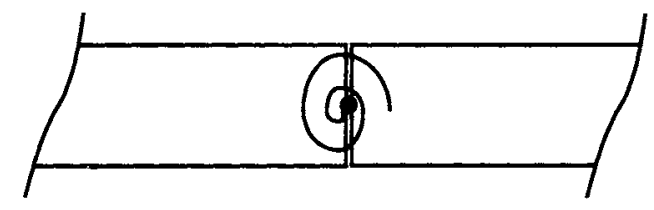

Pinned Joint at Crack Location

Figure 5. Simple crack models for beam elements. 
For illustration, the open crack will be modeled using plate elements. The geometry is modeled by removing elements where the crack is located. Figure 6 shows this in the case of plate elements, and shows the side view of the mesh used. Clearly more complex methods may be used, and the review papers quoted earlier give further details.

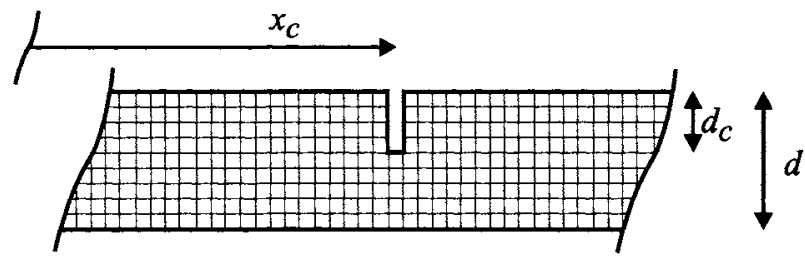

Figure 6. A simple crack model using plate elements.

The approach of Christides and Barr Clearly some of the material adjacent to the crack will not be stressed and thus will offer only a limited contribution to the stiffness. The actual form of this increased flexibility is quite complicated, but in this paper we approximate this phenomenon as a variation in the local flexibility. In reality, for a crack on one side of a beam, the neutral axis will change in the vicinity of the crack, but this will not be considered here. Shen and Pierre (1994); Carneiro and Inman (2001, 2002) have extended this approach to consider single edge cracks. Christides and Barr (1984) considered the effect of a crack in a continuous beam and calculated the stiffness, $E I$, for a rectangular beam to involve an exponential function given by

$$
E I(x)=\frac{E I_{0}}{1+C \exp \left(-2 \alpha\left|x-x_{c}\right| / d\right)}
$$

where $C=\left(I_{0}-I_{c}\right) / I_{c} . \quad I_{0}=\frac{w d^{3}}{12}$ and $I_{c}=\frac{w\left(d-d_{c}\right)^{3}}{12}$ are the second moment of areas of the undamaged beam and at the crack. $w$ and $d$ are the width and depth of the undamaged beam, and $d_{c}$ is the crack depth. $x$ is the position along the beam, and $x_{c}$ the position of the crack. $\alpha$ is a constant that Christides and Barr estimated from experiments to be 0.667 . The inclusion of the stiffness reduction of Christides and Barr (1984) into a finite element model of a structure, using beam elements, is complicated because the flexibility is not local to one or two elements, and thus the integration required to produce the stiffness matrix for the beam would have to be performed numerically every time the crack position changed. Furthermore, for complex structures, without uniform long beams, Equation (3.1) would only be approximate. Sinha et al. (2002) used a simplified approach, where the stiffness reduction of Christides and Barr was approximated by a triangular reduction in stiffness. An example of this approximation is shown in Figure 7, for a crack of depth $5 \%$, located at $x=0$. The advantage of this simplified model is that the stiffness reduction is now local, and the stiffness matrix may be written as an explicit function of the crack location and depth. For cracks of small depth a good approximation to the length of the beam influenced by the crack is $2 d / \alpha$. 


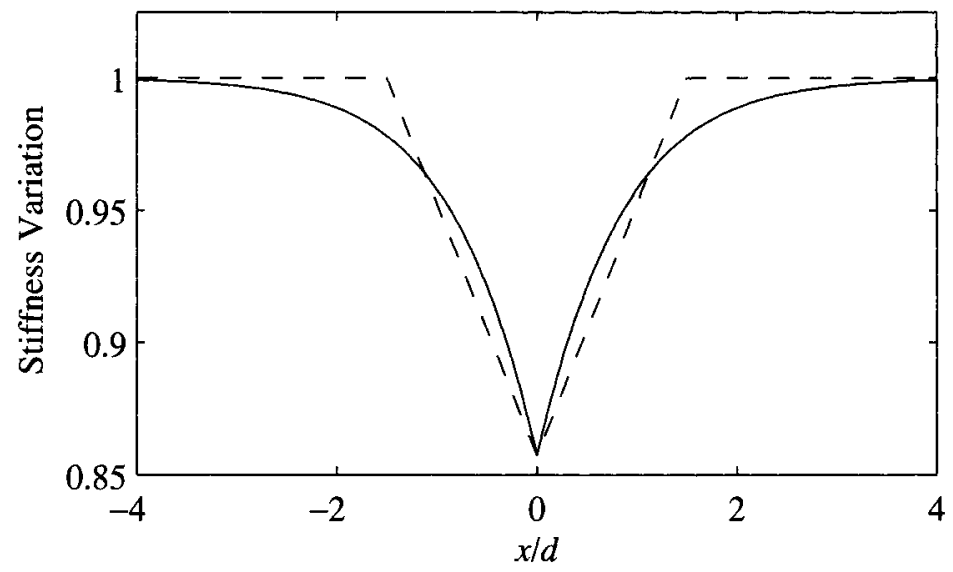

Figure 7. The variation in beam stiffness for the approaches of Christides and Barr (1984) (solid line) and Sinha et al. (2002) (dashed line).

Fracture mechanics approach An alternative approach is to estimate the increased flexibility caused by the crack, using empirical expressions of stress intensity factors from fracture mechanics. Lee and Chung (2001) gave such an approach based on the relationships given by Tada et al. (1973). Only a summary of the relevant equations will be given here. The element stiffness matrix is given by

$$
\mathbf{K}_{c}=\mathbf{T}^{\top} \mathbf{C}^{-1} \mathbf{T}
$$

where the transformation, $\mathbf{T}$, is

$$
\mathbf{T}=\left[\begin{array}{cccc}
-1 & 0 & 1 & 0 \\
0 & -1 & 0 & 1
\end{array}\right]
$$

The flexibility matrix, $\mathbf{C}$, for an element containing the crack in the middle, is given by

$$
\mathbf{C}=\frac{1}{6 E I}\left[\begin{array}{cc}
2 \ell_{e}^{3} & 3 \ell_{e}^{2} \\
3 \ell_{e}^{2} & 6 \ell_{e}
\end{array}\right]+\frac{18 \pi\left(1-\nu^{2}\right)}{E w d^{2}}\left[\begin{array}{cc}
\ell_{e}^{2} & 2 \ell_{e} \\
2 \ell_{e} & 4
\end{array}\right] \int_{0}^{d_{c} / d} \beta F_{I}^{2}(\beta) \mathrm{d} \beta
$$

where $\ell_{e}$ is the element length and $\nu$ is Poisson's ratio. $F_{I}(\beta)$ is the correction factor for the stress intensity factor, and may be approximated as

$$
F_{I}(\beta)=\sqrt{\frac{\tan (\pi \beta / 2)}{\pi \beta / 2}} \frac{0.923+0.199[1-\sin (\pi \beta / 2)]^{4}}{\cos (\pi \beta / 2)} .
$$

This formulation does give the stiffness matrix of the element containing the crack explicitly in terms of the crack depth. There are two difficulties with using this approach for structural health monitoring. The main problem is that the crack is located at the centre of the element, requiring that the finite element mesh be redefined as the crack moves. Furthermore the stiffness matrix of the crack is a complicated function of the crack depth, and does not depend on the crack location explicitly. 
A numerical comparison of the models The approaches to crack modelling will be compared using a simple example of a steel cantilever beam $1 \mathrm{~m}$ long, with cross section $25 \times 50 \mathrm{~mm}$. Bending in the more flexible plane is considered. The crack is assumed to be located at a distance $200 \mathrm{~mm}$ from the fixed end, and has a constant depth of $10 \mathrm{~mm}$ across the beam width.

The beam is modelled using 20 Euler-Bernoulli beam elements, and gives the natural frequencies shown in Table 3. For the plate elements the length is split into 401 elements and the depth into 10 elements. Thus the elements are approximately $2.5 \mathrm{~mm}$ square. A large number of elements is required because an element with linear shape functions is used. Table 3 shows the estimated natural frequencies using the Quad4 element in the Structural Dynamics Toolbox (Balmes, 2000).

Table 3. Natural frequencies (in $\mathrm{Hz}$ ) for the undamaged beam.

\begin{tabular}{|cc|c|c|}
\hline & & Beam & Plate \\
\hline Number DoF & & 40 & 13233 \\
\hline \multirow{4}{*}{ Modes } & 1 & 20.709 & 20.707 \\
& 2 & 129.78 & 129.39 \\
& 3 & 363.40 & 360.62 \\
& 4 & 712.16 & 701.96 \\
& 5 & 1177.4 & 1150.6 \\
\hline
\end{tabular}

The damaged beam was also modelled using the approaches discussed earlier, and the results are shown in Table 4 . The beam models all contain 20 elements, and the nodes are arranged such that the crack occurs in the middle of an element. Of course in the case of the discrete rotational spring a node is placed at the crack location. The reduction in the element stiffness is adjusted so that the percentage change in the first natural frequency is the same as that for the plate model. The other beam models are adjusted in a similar way. In the plate model, the crack is simulated by removing 4 elements and thus represents a saw cut $10 \mathrm{~mm}$ deep. The row of elements below the crack is also made thinner, so that the crack has negligible width. The differences in the lower natural frequencies are very similar for all models, and these differences are smaller than the changes that would occur due to small modelling errors, or changes due to environmental effects. Of course the accuracy at higher frequencies becomes less since the modes are influenced more by local stiffness variations.

Comparison with experimental results The previous section has shown that the natural frequencies predicted from different models are very close. Of course the question is whether the differences in these predictions are smaller than the measurement errors. As a demonstration the example of Rizos et al. (1990) will be used. Kam and Lee (1992) and Lee and Chung (2001) also used these results. The example is a steel cantilever beam of cross-section $20 \times 20 \mathrm{~mm}$ and length $300 \mathrm{~mm}$. Table 5 shows the measured and predicted frequencies of the uncracked beam. Rizos et al. (1990) propagated cracks at a number of different positions and depths, but here only a crack $80 \mathrm{~mm}$ from the cantilever root, and depths of 2 and $6 \mathrm{~mm}$ will be considered. Table 5 also shows the measured 
Table 4. The percentage changes in the natural frequencies for the damaged beam.

\begin{tabular}{|c|c|c|c|c|c|c|}
\hline & \multicolumn{4}{|c|}{ Beam } & \multirow[t]{2}{*}{ Plate } \\
\hline & & $\begin{array}{c}\text { Element Stiffness } \\
\text { Reduction }\end{array}$ & $\begin{array}{c}\text { Discrete } \\
\text { Spring }\end{array}$ & $\begin{array}{l}\text { Sinha et } \\
\text { al. }(2002)\end{array}$ & $\begin{array}{c}\text { Lee and } \\
\text { Chung (2001) }\end{array}$ & \\
\hline \multirow{5}{*}{ Modes } & 1 & 4.18 & 4.18 & 4.18 & 4.18 & 4.18 \\
\hline & 2 & 0.07 & 0.04 & 0.08 & 0.04 & 0.04 \\
\hline & 3 & 1.24 & 1.23 & 1.24 & 1.20 & 1.22 \\
\hline & 4 & 2.99 & 3.08 & 2.98 & 2.99 & 3.07 \\
\hline & 5 & 2.37 & 2.45 & 2.37 & 2.34 & 2.69 \\
\hline
\end{tabular}

natural frequencies for these crack depths. The damaged cantilever beam is modelled using the beam methods described earlier. The depth of the crack is optimised so that the percentage change in the first mode matches the experimental result, to allow for possible errors in measuring the crack depth. Tables 6 and 7 show the measured and predicted frequency changes for the 2 crack depths. The results clearly show that the differences in the natural frequencies predicted by the models are smaller than the measurement errors. Thus the simple models for cracked beams may be used with confidence in health monitoring applications.

Table 5. The natural frequencies (in $\mathrm{Hz}$ ) for the experimental cantilever beam example.

\begin{tabular}{|cc|c|c|c|c|}
\hline & & FE Model & \multicolumn{3}{|c|}{ Experimental } \\
& & Undamaged & Undamaged & 2 mm Crack & $6 \mathrm{~mm}$ Crack \\
\hline \multirow{3}{*}{ Modes } & 1 & 185.1 & 185.2 & 184.0 & 174.7 \\
& 2 & 1159.9 & 1160.6 & 1160.0 & 1155.3 \\
& 3 & 3247.6 & 3259.1 & 3245.0 & 3134.8 \\
\hline
\end{tabular}

Table 6. The percentage changes in the natural frequencies for the damaged beam with a $2 \mathrm{~mm}$ crack.

\begin{tabular}{|cc|c|c|c|c|c|}
\hline & $\begin{array}{c}\text { Element Stiffness } \\
\text { Reduction }\end{array}$ & $\begin{array}{c}\text { Discrete } \\
\text { Spring }\end{array}$ & $\begin{array}{c}\text { Sinha et } \\
\text { al. (2002) }\end{array}$ & $\begin{array}{c}\text { Lee and } \\
\text { Chung (2001) }\end{array}$ & $\begin{array}{c}\text { Experi- } \\
\text { mental }\end{array}$ \\
\hline \multirow{3}{*}{ Modes } & 1 & 0.648 & 0.648 & 0.648 & 0.648 & 0.648 \\
& 2 & 0.065 & 0.063 & 0.130 & 0.063 & 0.052 \\
& 3 & 0.606 & 0.610 & 0.604 & 0.606 & 0.433 \\
\hline
\end{tabular}

\subsection{Composite Structures}

Composite structures have an excellent performance, although this deteriorates significantly with damage. Unfortunately damage, due to impact events for example, are difficult to detect visually, and hence some method of non-destructive testing of these structures is required. Zou et al. (2000) reviewed the vibration based methods that are available to monitor composite structures. Since this paper considers inverse methods for 
Table 7. The percentage changes in the natural frequencies for the damaged beam with a $6 \mathrm{~mm}$ crack.

\begin{tabular}{|cc|c|c|c|c|c|}
\hline & & $\begin{array}{c}\text { Element Stiffness } \\
\text { Reduction }\end{array}$ & $\begin{array}{c}\text { Discrete } \\
\text { Spring }\end{array}$ & $\begin{array}{c}\text { Sinha et } \\
\text { al. (2002) }\end{array}$ & $\begin{array}{c}\text { Lee and } \\
\text { Chung (2001) }\end{array}$ & $\begin{array}{c}\text { Experi- } \\
\text { mental }\end{array}$ \\
\hline \multirow{3}{*}{ Modes } & 1 & 5.67 & 5.67 & 5.67 & 5.67 & 5.67 \\
& 2 & 0.56 & 0.54 & 0.88 & 0.54 & 0.46 \\
& 3 & 4.92 & 4.95 & 4.49 & 4.92 & 3.81 \\
\hline
\end{tabular}

damage estimation, this section will only consider the parameterisation of the damage in composite structures, and in particular the modelling of delaminations. Although composite structures have other modes of failure, such as matrix cracking, fibre breakage or fibre-matrix debonding (Ostachowicz and Krawczuk, 2001), these damage mechanisms produce similar changes in the vibration response to that obtained for damage in metallic structures. However delamination is a serious problem in composite structures, and has no parallel to damage mechanisms in other materials. Once the damage is parameterised then inverse methods, such as sensitivity analysis, may be applied.

Zou et al. (2000) reviewed methods to model delaminations, and here we will concentrate on simple models. For example, if a structure is modelled with beam or plate elements, then only beam or plates elements should be used to model the structure with delaminations. Delamination occurs when adjacent plies in a laminated composite debond. For beam structures the simpliest case of a through width delamination, parallel to the beam surface, was modelled using four beam segments (Majumdar and Suryanarayan, 1988; Tracy and Pardoen, 1989). Separate beam elements were used above and below the delamination, and the constraints to join these elements to those of the undamaged parts of the beam needed to be applied carefully. Zou et al. (2000) detailed further development of these models. One difficulty with using these models for parameter based identification is that changing the length and position of a delamination requires the model to be remeshed, and care must be exercised in calculating the associated sensitivity matrices. The techniques detailed by Sinha et al. (2002) for the position of cracks might be extended to this case. Paolozzi and Peroni (1990) highlighted that the most sensitive modes are those whose wavelength is approximately the same size as the delamination. Luo and Hanagud (1995) used a sensitivity based method to detect delaminations, and they also discovered that some modes split to give two closely spaced natural frequencies.

\subsection{Joint Models and Generic Elements}

One major difficulty in parametric approaches is that a model is required that accurately reflects the effect of damage on the mass and stiffness matrices. To some extent the situation is helped when low frequency vibration measurements are used because any local stiffness reduction will have a very similar effect on the dynamic response. Thus it is possible to use equivalent parameters, such as element stiffnesses, to model the damage. Generic elements (Gladwell and Ahmadian, 1995; Friswell et al., 2001) take this approach further by allowing changes to the eigenvalues and eigenvectors of the stiffness matrices 
of structural elements or substructures. These changes are usually constrained so that properties such as the rigid body modes and the geometric symmetry are retained.

Generic elements introduce flexibility into the joint in a controlled way. Other equivalent models, such as discrete rotational springs, offset parameters or changing element properties may also be used, although generic parameters do have advantages (Friswell et al., 2001). In particular, all models prejudge how the damage will affect the full model of the structure, whereas the generic element approach automatically finds the likely low frequency motion of the joint. Consider a two dimensional $\mathrm{T}$ joint constructed from three beam elements. Each node has three degrees of freedom and, since the substructure has four nodes, the substructure stiffness matrix has three rigid body eigenvectors and nine flexible eigenvectors (Titurus et al., 2003a). The lower eigenvectors have much simpler deformation shapes that are more likely to represent the motion the substructure would undergo in many of the global modes of the structure. Thus reducing the eigenvalues corresponding to these eigenvectors makes the joint substructure more flexible in the frequency range of the global dynamics, and may be used to model damage. Higher frequency eigenvectors of the substructure may also be included if the motion of the joint is more complex, however the lower eigenvectors of the joint are likely to adequately characterise the low frequency dynamics of the structure.

Generic elements have been developed for use in model updating and may be considered as equivalent models of elements or substructures (Gladwell and Ahmadian, 1995). Law et al. (2001) applied generic elements to the finite element model updating of the Tsing Ma bridge in Hong Kong. Wang et al. (1999) used generic elements in damage detection, dealing with the simulated problem of damage detection in a frame structure with flexible L-shaped and T-shaped structural joints.

The form of generic element parameterisation assumes that the damage only influences the stiffness properties and that the mass properties are modelled correctly. Thus only changes in the stiffness matrices are allowed. The eigenvalue problem for any selected sub-structure or element stiffness matrix can be written as

$$
\left(\mathbf{K}^{\mathrm{SUB}}-\lambda_{i} \mathbf{I}\right) \phi_{i}=\mathbf{0}, \quad\left(\boldsymbol{\Phi}^{\mathrm{SUB}}\right)^{\top} \mathbf{K}^{\mathrm{SUB}} \boldsymbol{\Phi}^{\mathrm{SUB}}=\left[\begin{array}{cc}
\mathbf{0} & \mathbf{0} \\
\mathbf{0} & \boldsymbol{\Lambda}_{S}
\end{array}\right]
$$

where

$$
\boldsymbol{\Phi}^{\mathrm{SUB}}=\left[\phi_{1}, \ldots, \phi_{n_{R}}, \phi_{n_{R}+1}, \ldots, \phi_{n_{S U B}}\right]=\left[\boldsymbol{\Phi}_{R}, \boldsymbol{\Phi}_{S}\right] \in \Re^{n_{S U B} \times n_{S U B}},
$$

and $n_{R} \leq 6 . \mathbf{K}^{\mathrm{SUB}}$ is a sub-structure stiffness matrix, $\boldsymbol{\Phi}^{\mathrm{SUB}}$ is the eigenvector matrix of $\mathbf{K}^{\mathrm{SUB}}, \lambda_{i}$ and $\phi_{i}$ are the $i$ th eigenvalue and eigenvector of matrix $\mathbf{K}^{\mathrm{SUB}}$, respectively. Sub-matrix $\boldsymbol{\Lambda}_{S}$ is a diagonal matrix of non-zero eigenvalues of matrix $\mathbf{K}^{\mathrm{SUB}}$. The dimensions of these matrices depend on the size of the chosen sub-structure, where $n_{\text {SUB }}$ is a number of degrees of freedom of substructure and $n_{R} \leq 6$ is the number of rigid body modes, $\boldsymbol{\Phi}_{R}, \boldsymbol{\Phi}_{S}$ are sub-matrices of $\boldsymbol{\Phi}^{\text {SUB }}$ corresponding to the rigid and structural modes, respectively.

A modified set of sub-structure eigenvectors may be obtained by a linear transformation, as

$$
\left[\boldsymbol{\Phi}_{0 R}, \boldsymbol{\Phi}_{0 S}\right]=\left[\boldsymbol{\Phi}_{R}, \boldsymbol{\Phi}_{S}\right]\left[\begin{array}{cc}
\mathbf{S}_{R} & \mathbf{S}_{R S} \\
\mathbf{0} & \mathbf{S}_{S}
\end{array}\right]
$$


where the index 0 denotes the original quantities and matrices without index 0 represent modified quantities. Notice that in Equation (3.8) the modified rigid body modes do not contain any of the structural modes. By rearranging Equation (3.6) and using Equation (3.8), the modified sub-structure stiffness matrix may be written as

$$
\mathbf{K}^{\mathrm{SUB}}=\boldsymbol{\Phi}_{0 S} \mathbf{S}_{S}^{\top} \boldsymbol{\Lambda}_{S} \mathbf{S}_{S} \boldsymbol{\Phi}_{0 S}^{\top}=\boldsymbol{\Phi}_{0 S}\left[\begin{array}{ccc}
\kappa_{1,1} & \cdots & \kappa_{1,\left(n_{S U B}-n_{R}\right)} \\
& \ddots & \vdots \\
\mathrm{SYM} & & \kappa_{\left(n_{S U B}-n_{R}\right),\left(n_{S U B}-n_{R}\right)}
\end{array}\right] \boldsymbol{\Phi}_{0 S}^{\top} \text {. }
$$

Equation (3.9) is the basis for generic element parameterisation for damage detection. $\kappa_{1,1}, \ldots, \kappa_{\left(n_{S U B}-n_{R}\right),\left(n_{S U B}-n_{R}\right)}$ are the most general parameters for this parameterisation. Employing additional assumptions related to the geometric symmetry or antisymmetry of the corresponding eigenvectors will significantly reduce the total number of parameters. The sensitivity of natural frequencies with respect to these parameters is

$$
\frac{\partial \lambda_{i}}{\partial x_{j}}=\phi_{i}^{\top} \frac{\partial}{\partial x_{j}}\left(\mathbf{K}_{0}+\sum_{l=1}^{N_{P}} \mathbf{K}_{l}\left(\mathbf{x}_{l}\right)\right) \phi_{i}=\phi_{i}^{\top} \frac{\partial \mathbf{K}_{r}\left(\mathbf{x}_{r}\right)}{\partial x_{j}} \phi_{i}
$$

where $N_{P}$ is a number of parameterised substructures or elements, $\mathbf{x}_{l}$ is a group of parameters corresponding to $l$ th substructure or element with corresponding stiffness matrix $\mathbf{K}_{l}, \mathbf{x}$ is a vector of all parameters, $\mathbf{K}_{0}$ is non-parameterised part of the global stiffness matrix, $\lambda_{i}$ is the $i$ th eigenvalue and $x_{j}$ is $j$ th parameter of a chosen parameterisation that is associated with the $r$ th substructure or element.

Consider a two dimensional $T$ joint constructed from three beam elements. Each node has three degrees of freedom and, since the substructure has four nodes, the substructure stiffness matrix has three rigid body eigenvectors and nine flexible eigenvectors. Figure 8 shows the nine flexible eigenvectors for this substructure, where the circles and dots represent the nodes and the dotted line is the undeformed joint. The finite element shape functions have been used to produce smooth deformation shapes. The lower eigenvectors have much simpler deformation shapes that are more likely to represent the motion the substructure would undergo in many of the global modes of the structure. Thus reducing the eigenvalues corresponding to these eigenvectors makes the joint substructure more flexible in the frequency range of the global dynamics. Higher frequency eigenvectors of the substructure may also be included if the motion of the joint is more complex, however the first two eigenvectors of the $\mathrm{T}$ joint were found to characterise the dynamics of the frame structure considered later. Gladwell and Ahmadian (1995) gave further explanation of the physical meaning of generic elements.

\subsection{Distributed Damage}

Teughels et al. (2002) presented a sensitivity-based finite element updating method for damage assessment that minimised differences between the experimental and predicted modal data. The parameterisation of the damage (both localisation and quantification) was represented by a reduction factor of the element bending stiffness. The number of unknown variables was reduced to obtain a physically meaningful result, by using a set of 


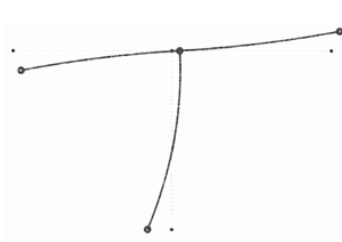

Eigenvector 1

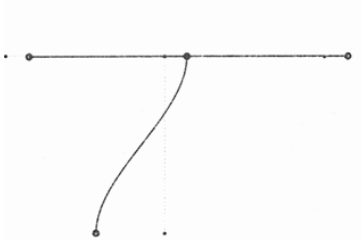

Eigenvector 4

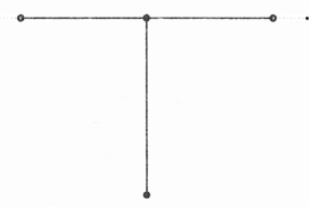

Eigenvector 7

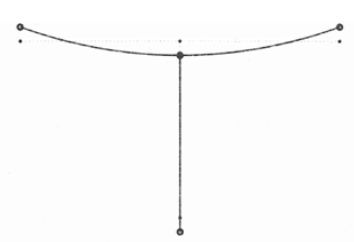

Eigenvector 2

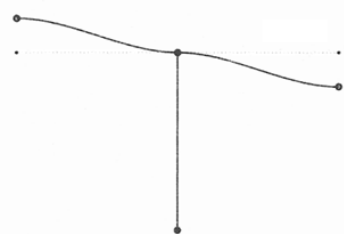

Eigenvector 5

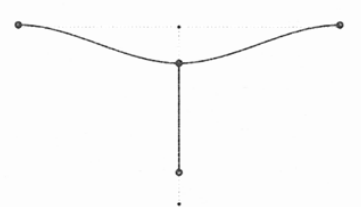

Eigenvector 8

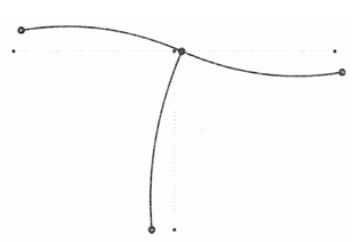

Eigenvector 3

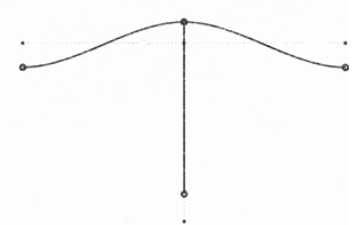

Eigenvector 6

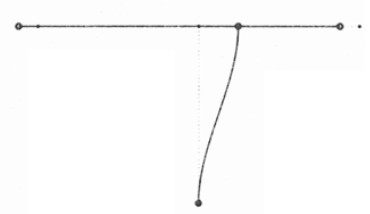

Eigenvector 9

Figure 8. Substructure eigenvectors for a $\mathrm{T}$ joint.

damage functions to determine the spatial bending stiffness distribution. The updating parameters were then the multiplication factors of the damage functions. The procedure was illustrated on a reinforced concrete beam and on a highway bridge (Teughels and Roeck, 2004).

\section{An Example of Subset Selection using Generic Elements}

The proposed strategy is evaluated on a structure consisting of four thin-walled tubes connected to each other by four fillet welds. These joints were intentionally manipulated to produce one healthy and six damage cases. Titurus et al. (2003a) gave a detailed discussion of the identification results for the healthy/undamaged structure and Titurus et al. (2003b) described the estimation of the damage cases. Figure 9 shows the experimental structure, and Figure 10 shows the discretisation and experimental (EMA) measurement locations (the response was measured at the $\mathrm{FE}$ nodes). The finite element (FEM) nodes were placed at the measurement locations. Thus 32 degrees of freedom were measured, whereas the FE model contained 96 degrees of freedom (three degrees of freedom per node). The in-plane dynamics of the structure were measured, and the structure was supported in the free-free condition by elastic bands. 


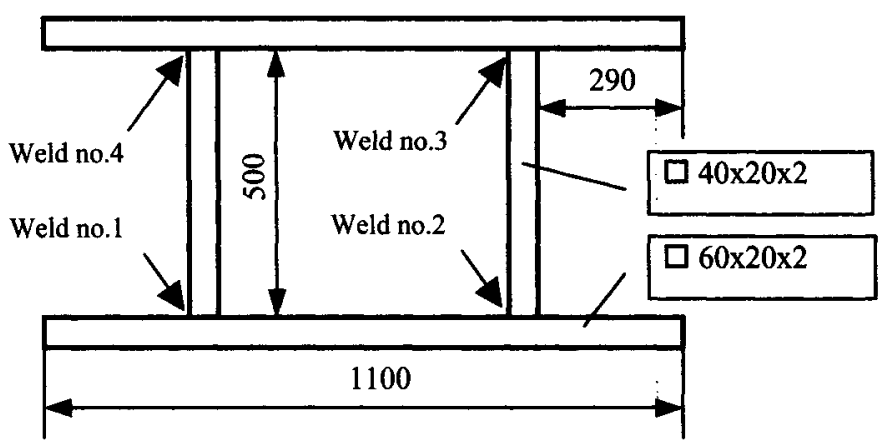

Figure 9. The outline of the $\mathrm{H}$-frame structure (dimensions in $\mathrm{mm}$ ).

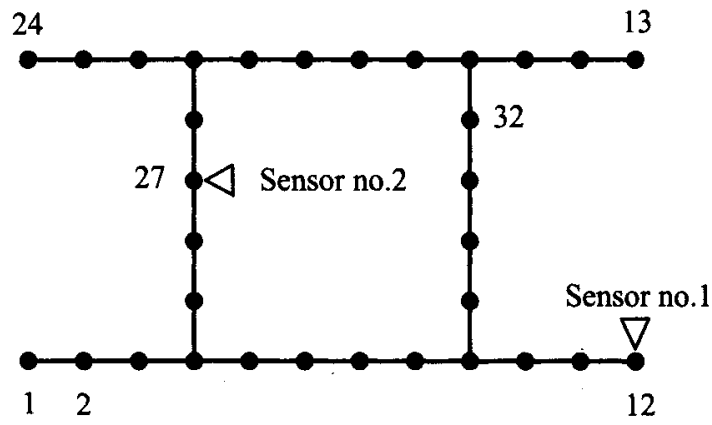

Figure 10. The discretisation of the H-frame structure.

\subsection{Damage cases}

Figure 11 gives a detailed description of all of the damage cases. These cases were produced by the intentional incompleteness of one or more of the fillet welds used to interconnect the four tubular parts of the structure. The shaded lines show the welds completed for each of the four joints, for each damage state. Note that State VII has all welds in place and hence is the undamaged structure. A distinctive feature of this structure is its geometrical symmetry, which is likely to cause problems for damage location based on measured natural frequencies alone. Two different approaches will be evaluated; the first assumes that the influence of the transducer mass will be sufficient to break the symmetry, whilst the second uses the measured mode shapes and their associated sensitivities. However, partial damage location will be tried, based on the use of natural frequencies alone.

These damage cases were selected to give a reasonable coverage of all possible combinations of damage cases, within practical constraints. This section concentrates on the 
single location damage case, and so only State VII, State VI and State V from Figure 11 will be studied in detail.

\begin{tabular}{|c|c|c|c|c|c|c|}
\hline State I & State II & State III & State IV & State $V$ & State VI & State VII \\
\hline $4 \quad 3$ & 4 & $4 \quad 3$ & 4 & & 4 & 4 \\
\hline 1 & 12 & 2 & 1 & 2 & 1 & 2 \\
\hline
\end{tabular}

Figure 11. An overview of the damage cases considered.

\subsection{Identification results for the damage cases}

A full modal test was performed for each of the damage cases shown in Figure 11, however as the number of results is large only a selection will be considered here. The measurements were performed in the frequency range from 0 to $625 \mathrm{~Hz}$. Table 8 gives the first nine measured natural frequencies for all of the damage cases. The fifth and sixth modes swap order between damage states III and IV. The last column corresponds to the undamaged/healthy structure, that is the structure with fully welded joints. Generally, the natural frequencies decrease with increasing level of damage, as a result of the decreasing stiffness of the structure. However, some small increases were observed in some natural frequencies from one case to another. One possible reason might be a small decrease in mass due to the absence of some weld material. Alternatively, taking the structure from the free-free suspension to undertake the welding may give small frequency changes due to slightly different suspension conditions.

Table 8. The natural frequencies (Hz) of the healthy (State VII) and damaged (State I to VI) structure. Note that modes 5 and 6 swap between States III and IV due to the damage.

\begin{tabular}{|c|c|c|c|c|c|c|c|}
\hline Mode & State I & State II & State III & State IV & State V & State VI & State VII \\
\hline 1 & 27.63 & 33.57 & 34.18 & 48.60 & 50.26 & 60.06 & 60.57 \\
2 & 118.64 & 120.94 & 120.74 & 125.04 & 124.82 & 126.60 & 126.53 \\
3 & 126.38 & 129.92 & 130.64 & 138.97 & 139.63 & 147.86 & 147.05 \\
4 & 169.77 & 172.28 & 172.32 & 174.77 & 175.42 & 175.86 & 175.89 \\
5 & 264.77 & 275.73 & 275.42 & 280.09 & 280.33 & 280.81 & 280.76 \\
6 & 279.64 & 280.38 & 280.24 & 300.44 & 301.72 & 319.60 & 320.56 \\
7 & 298.91 & 312.58 & 317.15 & 342.10 & 347.97 & 359.55 & 360.70 \\
8 & 393.61 & 396.78 & 399.06 & 418.68 & 420.05 & 436.24 & 437.72 \\
9 & 550.55 & 551.78 & 552.71 & 560.06 & 560.63 & 565.93 & 566.52 \\
\hline
\end{tabular}


Figure 12 shows the modal assurance criteria (MAC) matrices between the reference mode shapes of the healthy structure and mode shapes corresponding to the damaged cases. It is clear that the fifth and sixth modes interact and swap over between damage states I and VII. Another interesting feature shown by the MAC matrices is the relative insensitivity of the mode shapes to increasing damage, despite large changes in the natural frequencies. Titurus et al. (2003a) gave other experimental results, in particular the mode shapes corresponding to the healthy structure and further discussion of modelling issues.

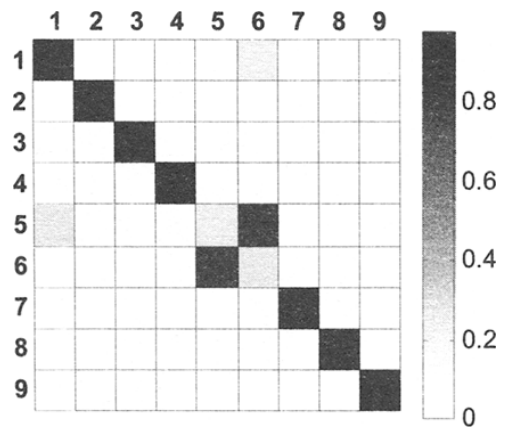

Figure 12. MAC criterion between State VII and damage State I. The rows of each MAC matrix correspond to mode shapes of State VII while columns correspond to mode shapes of State I.

\subsection{Parameterisation overview}

Section 3.3 provided a detailed explanation of parameterisations to be used for damage location, however, for the sake of completeness, a summary is provided here. Parameterisation $\mathrm{A}$ is expressed in terms of two groups of generic elements. The first group consists of one generic substructure that models the parts of the structure containing the fillet welds, and two parameters are required for each substructure, as shown in Figure 13. The other group consists of three different generic elements, each requiring one parameter, as shown in Figure 13. Thus, parameterisation $\mathrm{A}$ requires the parameter vector $\mathbf{x}$ given by

$$
\mathbf{x}=\left[x_{1}, x_{2}, x_{3}, x_{4}, x_{5}\right]^{\top}=\left[\kappa_{11}^{1}, \kappa_{22}^{1}, \kappa_{11}^{2}, \kappa_{11}^{3}, \kappa_{11}^{4}\right]^{\top}
$$

where $\kappa_{j k}^{i}$ denotes the $(j, k)$ element of the matrix of the $i$ th element/substructure, based on generic elements as detailed in Equation (3.9). The values of these parameters may be determined by model updating. This parameterisation allows partial localisation to the type of region where damage has occurred.

Parameterisation B allows similar elements or substructures to have independent values of the corresponding generic parameters, to enable complete damage localisation. Parameterisation $B$ requires 28 parameters for the $\mathrm{H}$-shaped structure, as shown in Fig- 

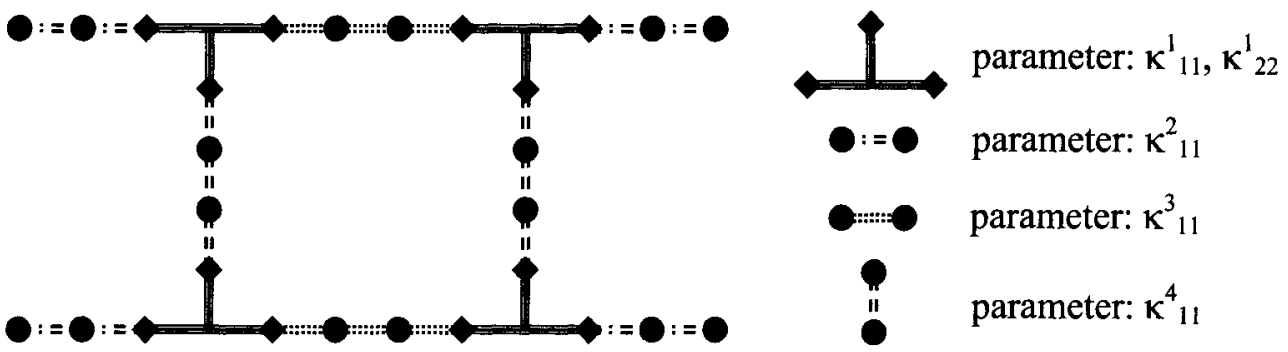

Figure 13. Parameterisation $\mathrm{A}$ of the baseline model of the thin-walled $\mathrm{H}$ structure.

ure 14, and these are defined as,

$$
\begin{array}{ll}
x_{2 i-1}=\kappa_{11}^{i}, \quad x_{2 i}=\kappa_{22}^{i}, & i=1,2,3,4, \\
x_{j+4}=\kappa_{11}^{j}, & j=5,6, \ldots, 24 .
\end{array}
$$

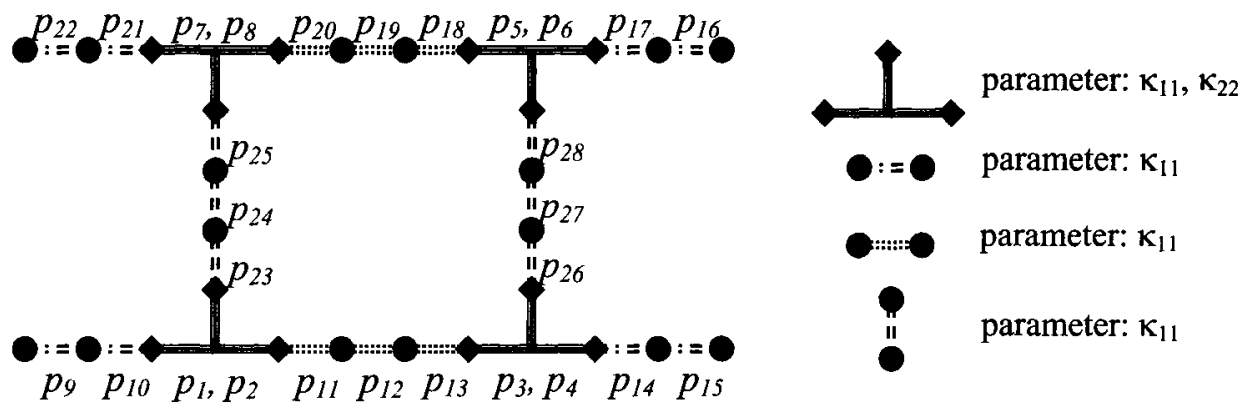

Figure 14. Parameterisation $B$ of the baseline model of the thin-walled $\mathrm{H}$ structure.

\subsection{Partial damage location - natural frequencies alone}

In this section, parameterisation $\mathrm{A}$ will be used for partial damage localisation, using only the measured natural frequencies. This simplified form of damage localisation is chosen as a first step in the damage detection of a geometrically symmetric structure. The first seven natural frequencies corresponding to the healthy and damaged structures, as well as the sensitivity matrix $\mathbf{S}$, determined at the updated parameters values (Titurus et al., 2003a), were used to test the subset selection approach proposed in Section 2.2. 
Both the sensitivity matrix and the measured frequency differences for the considered damage cases, were normalised by the corresponding measured natual frequencies. Since single-location damage states are of primary interest, State VI and State V will be used, as they represent two levels of damage in one fillet weld. State IV and State II will also be considered, as these are multi-location damage states with different levels of damage (see Figure 11).

The results of damage location, in the form of subspace angles, are shown in Figure 15. Individual groups of columns correspond to particular model parameters. Within each group, corresponding to each parameter, the columns represent different comparisons of the damage cases (State II, IV, V, VI) with the healthy structure (State VII). Figure 15 suggests that the damage corresponds to parameter $x_{1}=\kappa_{11}^{1}$, which corresponds to the generic substructures containing the welded joints. Thus the damage is correctly localised to the welded joint. An important feature of this study is that even relatively small damage corresponding to State VI is readily observable and clearly identifiable. An increasing level of damage, represented here by State V, leads to improved and clearer identification of damage location or damage type.

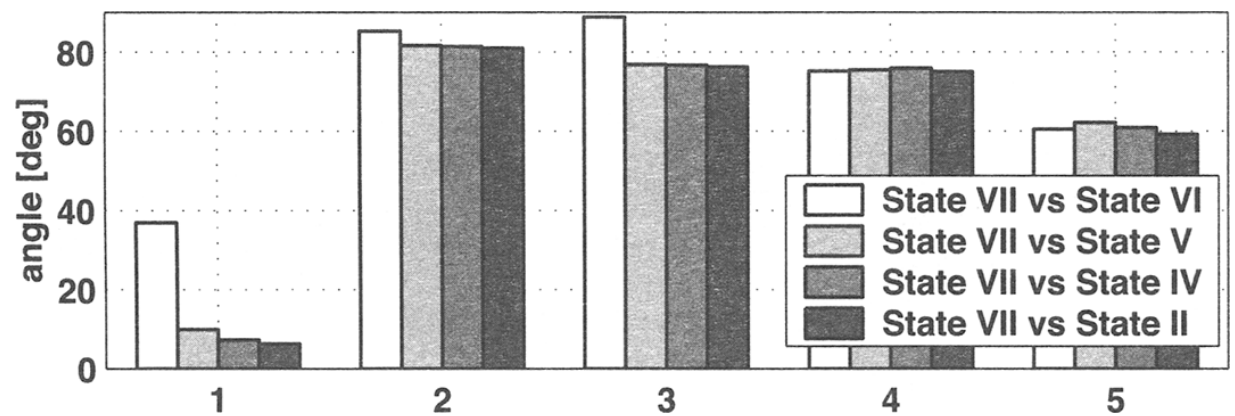

Figure 15. Subspace angles for partial localisation, parameterisation A.

The results disagree with the expectation that the increasing level of damage should lead to increasing subspace angles and consequently to a deteriorating quality of damage localisation, due to the increasing error in the linearisation of the original non-linear problem. However the level of damage for State VI is relatively small (the maximum difference between State VII and State VI is $-0.83 \%$ for the first natural frequency, see Table 8) and therefore susceptible to measurement error. State $V$ is characterised by a larger extent of damage, and therefore large differences in the natural frequencies (the maximum difference for this combination is $-17.02 \%$ for the first natural frequency), produces smaller subspace angles. Figure 15 also gives the subspace angles for State II and State IV, and the angles corresponding to parameter 1 are smaller still. Although these are multi-damage cases, the damage still lies in the joints. Another noticeable and beneficial feature of the results is the insensitivity of the subspace angles from other parameters due to damage in the welded joints, reducing the possibility of false alarms.

Figure 16 provides additional information in terms of a selection tree. A selection tree is a representation of the forward parameter subset selection where each node of the 
tree corresponds to a selected subset and its colour represents the numerical value of the residual. The root of the tree represents the initial system, Equation (4.2). The branching factor and the depth of the tree are decided in advance and in our case results in binary selection trees with three levels corresponding to the selected parameter subsets. All three figures are determined using the first seven natural frequencies. The second best single parameter would be $x_{5}$, a parameter that also effectively monitors the stiffness in the regions connected with welded joints. The important relative indicators of damage level for a given situation are the absolute values of the residuals provided by the amplitude bar on the left of the Figure 16, as the ability to reproduce the measurement vector decreases with increasing damage level and consequently the magnitude of residuals also increases.

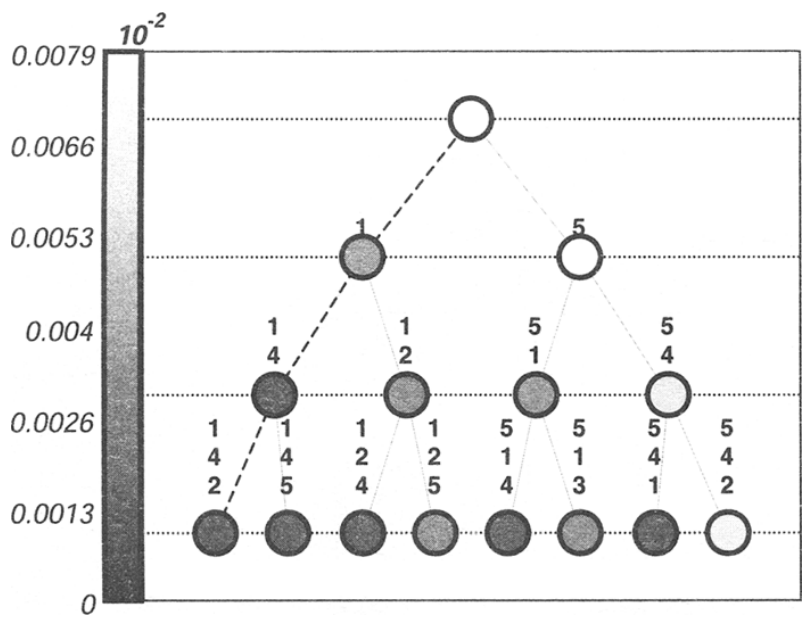

Figure 16. Binary tree representing forward selection, State VII vs. State VI.

\subsection{Complete damage detection - natural frequencies and mode shapes}

The only way to deal with damage localisation for geometrically symmetric structures is to use spatial information in the form of mode shapes. Once again the analysis was limited to the single location damage case, i.e. State VII (healthy), State VI (level 1 damage at welded joint 4, see Figure 9) and State V (level 2 damage at welded joint 4). The subspace angles corresponding to the individual parameters (parameterisation B) were computed by the techniques presented in Section 2.2. However, since the vector $\delta \mathbf{z}$ and the sensitivity matrix $\mathbf{S}$ contain elements corresponding to both the natural frequencies and the mode shapes, additional weighting must be included to represent the relative importance of the natural frequency and mode shape information, as presented in Section 2.2.

There are problems in using mode shape information, particularly since the accuracy of their estimation from measured data is worse than for natural frequencies. This is 
compounded since the proposed approach uses the differences between the measured damaged and measured undamaged mode shapes. Thus only mode shapes that are sensitive to the candidate damage sites should be chosen. Table 8 shows the changes in the natural frequencies for the different damage states, and gives a good indication of this sensitivity. However the table shows the sensitivity of the natural frequencies, which is not necessarily the same as the sensitivity of the mode shapes. Certainly, if the natural frequencies change very little with damage, then the corresponding mode shapes will not be sensitive. Thus, of the first seven modes, modes $2,3,4$ and 5 are unlikely to give useful spatial information (note that mode 3 has been excluded because of the slight increase in the natural frequency in State VI). The sensitivity of the mode shapes to damage also increases with mode number, as the mode shapes corresponding to higher frequencies, contain more local deformation. Mode 1 is a global mode and therefore its shape is insensitive to damage.

The proposed approach using mode shapes will be demonstrated using spatial information from mode 6 . Relative errors in the first seven natural frequencies and the difference in the mode shape elements were used. No further weighting was included, as similar results were obtained with other weighting values. Figure 17 shows the subspace angles corresponding to damage State $\mathrm{V}$, and provides the correct indication of damage location, corresponding to parameter $x_{7}$. This parameter belongs to the fourth generic $\mathrm{T}$ substructure, representing fillet weld number 4 (see Figure 14). Other significant parameters indicated by the subspace angles are parameters $x_{23}$ to $x_{28}$, which are located on the crossbar neighbouring the damaged region. However the results from the frequency only estimation clearly indicated that the damage is located in the joints. Thus damage in welded joint 4 may be confidently predicted.

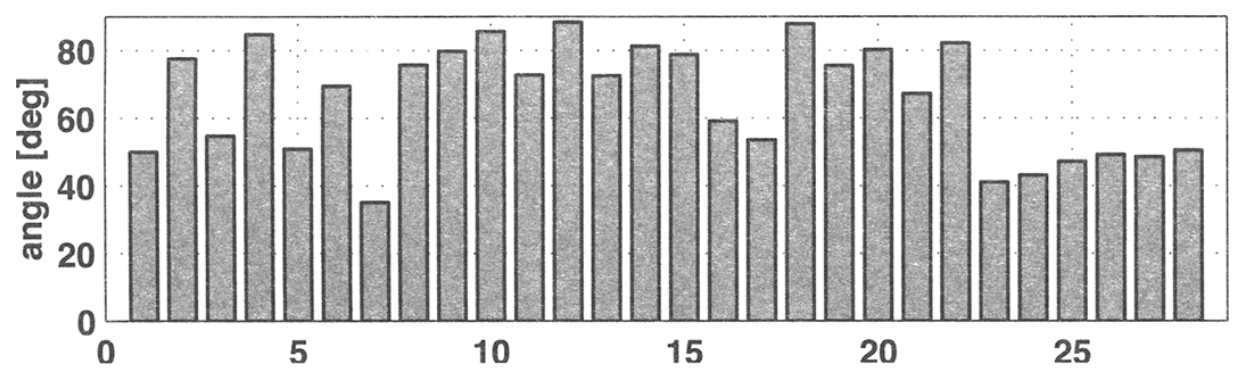

Figure 17. Subspace angles corresponding to State V, using seven natural frequencies and mode shape 6 .

\section{Methods using Mode Shapes}

This section considers a different approach where changes in the measured modes shapes are used directly to detect and locate damage. Farrar and Jauregui (1998a,b) compared several of these methods, such as the damage index method (Stubbs et al., 1992), mode 
shape curvatures (Pandey et al., 1991), the change in flexibilities (Pandey and Biswas, 1994) or the change in stiffness (Zimmerman and Kaouk, 1994). The example used was a road bridge with a concrete deck and steel supports. Different levels of damage were introduced, but the damage was only clearly located with most methods at the most severe level where the first natural frequency changed by over $7 \%$, and the mode shapes changed significantly. The damage index method (basically a measure of changes in modal strain energy) was found to be the most promising. Other methods based on pattern recognition, often using neural networks, are also popular (Sohn et al., 2001, 2002; Trendafilova and Heylen, 2003). These methods essentially provide curve fits using interpolation functions and are not based on physical models. The lack of a physical model also limits the scope for damage prognosis.

One of the major problems with methods using mode shapes is the incompleteness of the mode shapes. The number of measured degrees of freedom is always far smaller that the number of analytical degrees of freedom. Thus the mode shapes must be expanded or the analytical model must be reduced. In either case errors will be introduced because the model without damage is generally used for this reduction or expansion. The other source of incompleteness is the measured frequency range, which means that only a small number of modes will be measured. If damage causes a change in the stiffness matrix, this is further complicated because the high frequency modes have most effect on the elements of the stiffness matrix, but the lower frequency modes are generally measured. A huge number of different methods have been proposed and only a selection of the available methods will now be described.

\subsection{COMAC}

Perhaps the simplest method is the Coordinate Modal Assurance Criterion (COMAC). The usual Modal Assurance Criterion (MAC) correlates modes shapes by summing over the measured degrees of freedom. The COMAC sums over the modes and thus gives information about the correlation of the degrees of freedom (Lieven, 1988). If $\phi_{u i, j}$ is the $j$ th element of the $i$ th mode shape vector for the undamaged structure, and $\phi_{d i, j}$ is the corresponding quantity for the damaged structure, then the COMAC for degree of freedom $j$ is

$$
\operatorname{COMAC}_{j}=\frac{\left\|\sum_{i} \phi_{u i, j} \phi_{d i, j}\right\|^{2}}{\left\|\sum_{i} \phi_{u i, j} \phi_{u i, j}\right\|\left\|\sum_{i} \phi_{d i, j} \phi_{d i, j}\right\|} .
$$

Damage location is determined by those degrees of freedom with a low correlation between the healthy and damaged states. Note that local damage will affect all of the degrees of freedom to some extent, and so the changes in the mode shapes will not necessarily be local.

\subsection{Balancing the Eigenvalue Equation}

Suppose that the first $r$ natural frequencies and mode shapes are measured. If $\lambda_{d i}$ are the eigenvalues and $\phi_{d i}$ the mode shapes of the damaged structure, then

$$
\left[\lambda_{d i}^{2} \mathbf{M}_{d}+\lambda_{d i} \mathbf{D}_{d}+\mathbf{K}_{d}\right] \phi_{d i}=\mathbf{0}, \quad i=1, \ldots, r,
$$


where $\mathbf{M}_{d}, \mathbf{D}_{d}$ and $\mathbf{K}_{d}$ are the mass, damping and stiffness matrices of the damaged structure, which are of course unknown. Suppose that damage only affects the stiffness matrix, then $\mathbf{M}_{d}=\mathbf{M}_{u}, \mathbf{D}_{d}=\mathbf{D}_{u}$ and $\mathbf{K}_{d}=\mathbf{K}_{u}-\delta \mathbf{K}$ where $\mathbf{M}_{u}, \mathbf{D}_{u}$ and $\mathbf{K}_{u}$ are the mass, damping and stiffness matrices of the undamaged structure, which are assumed to be known. In practice model updating may be used to ensure that the model of the undamaged structure accurately represents the measured dynamics. $\delta \mathbf{K}$ is the unknown change in the stiffness matrix due to damage. Equation (5.2) then becomes,

$$
\mathbf{L}_{i}=\left[\lambda_{d i}^{2} \mathbf{M}_{u}+\lambda_{d i} \mathbf{D}_{u}+\mathbf{K}_{u}\right] \phi_{d i}=\delta \mathbf{K} \phi_{d i}, \quad i=1, \ldots, r
$$

In Equation (5.3) the vector $\mathbf{L}_{i}$ on the left side of the equation may be calculated. If the change in the stiffness matrix due to damage is local then only those parts of the stiffness matrix corresponding to the affected degrees of freedom will be non-zero, and hence the damage may be localised by the non-zero elements of the vector $\mathbf{L}_{i}$. If more than one mode is measured then the contribution from each mode may be averaged using the root mean square for each degree of freedom.

\subsection{Modal Strain Energy}

Zhang et al. (1998) presented a method which compared the modal strain energy within the elements. For element $j$, with corresponding element stiffness $\mathbf{K}_{j}$, the element modal strain energy ratio for the $i$ th mode $\left(S E R_{i j}\right)$ is

$$
S E R_{i j}=\frac{\phi_{i}^{\top} \mathbf{K}_{j} \phi_{i}}{\phi_{i}^{\top} \mathbf{K} \phi_{i}}=\frac{\phi_{i}^{\top} \mathbf{K}_{j} \phi_{i}}{\omega_{i}^{2}}
$$

where $\phi_{i}$ is the $i$ th mass normalised mode shape, $\omega_{i}$ is the $i$ th natural frequency and $\mathbf{K}$ is the stiffness matrix of the structure. A damage indicator $\beta_{i j}$ is then defined as the difference of the element modal strain energy ratio before and after damage as,

$$
\beta_{i j}=\frac{\phi_{d i}^{\top} \mathbf{K}_{j} \phi_{d i}}{\omega_{d i}^{2}}-\frac{\phi_{u i}^{\top} \mathbf{K}_{j} \phi_{u i}}{\omega_{u i}^{2}}
$$

where the subscripts $u$ and $d$ denote the healthy and damaged structures.

\subsection{Direct and Minimum Rank Update Methods}

Consider first the direct updating methods. The goal is often to reproduce the measured data (usually the modal model), by changing the stiffness matrix as little as possible (in some minimum norm sense). Historically these method were among the earliest in model updating (Friswell and Mottershead, 1995) and a number of generalisations have been proposed, depending on what is considered to be the reference quantities (Kenigsbuch and Halevi, 1998). A number of problems exist with the direct methods. There is no guarantee that the resulting matrices are positive definite (or semi-definite for structures with free-free modes), and extra modes may be introduced into the frequency range of interest. The standard methods do not enforce the connectivity of the structure, represented by the bandedness of the matrices and the pattern of zero terms, although Kabé 
(1985) gave a method that enforced the expected connectivity. More fundamental is that forcing the model to reproduce the data does not allow for the errors that will be present in the measured data. Mode shapes, in particular, can only be measured with a limited accuracy. The major problem for damage location, and indeed for error location in model updating, is that all elements in the matrices may be changed. If only a small number of sites are modelled incorrectly (or are damaged) then only a small number of the matrix elements will be changed. Generally, because of the minimum norm optimisation in the updating method, all the matrix elements would be changed a little, rather than a small number of elements changed substantially. Thus the effect of any damage present would be spread over all the degrees of freedom making location difficult.

Zimmerman and Kaouk (1994) and Kaouk and Zimmerman (1994) proposed that the change in the stiffness matrix should be low rank. This does not ensure that the change in stiffness will be local, as the stiffness change could be global but low rank. The method requires the rank of the stiffness change to be less than or equal to the number of measured modes used in the update. Zimmerman et al. (1995) gave an overview of this approach, and discussed issues such as the number of measured modes to use. Doebling (1996) extended the method by updating the elemental parameter vector rather than the global stiffness matrix. Abdalla et al. (1998, 2000) developed methods by minimising the change in the stiffness matrix, while enforcing constraints such as symmetry, sparsity and positive definiteness.

The development begins by combining Equation (5.3) for all $r$ measured modes to give,

$$
\delta \mathbf{K} \mathbf{V}_{d}=\mathbf{M}_{u} \mathbf{V}_{d} \Lambda_{d}^{2}+\mathbf{D}_{u} \mathbf{V}_{d} \Lambda_{d}+\mathbf{K}_{u} \mathbf{V}_{d}=\mathbf{B}
$$

where $\mathbf{V}_{d}=\left[\begin{array}{lll}\phi_{d 1} & \phi_{d 2} \ldots \phi_{d r}\end{array}\right]$ and $\Lambda_{d}=\operatorname{diag}\left[\lambda_{d 1} \lambda_{d 2} \ldots \lambda_{d r}\right]$

It may be proved (Zimmerman and Kaouk, 1994; Kaouk and Zimmerman, 1994) that the minimum rank of $\delta \mathbf{K}$ is $r$, and that this minimum rank solution to Equation (5.6) is

$$
\delta \mathbf{K}=\mathbf{B}\left[\mathbf{B}^{\top} \mathbf{V}_{d}\right]^{-1} \mathbf{B}^{\top}
$$

\subsection{Change in Flexibility}

The flexibility matrix is the inverse of the stiffness matrix. In terms of the mode shapes the flexibility matrix $\mathbf{C}$ is

$$
\mathbf{C}=\sum_{i=1}^{n} \frac{1}{\omega_{i}^{2}} \phi_{i} \phi_{i}^{\top}
$$

where $\lambda_{i}$ and $\phi_{i}$ are the $i$ th natural frequency and mass normalised mode shape and $n$ is the number of degrees of freedom in the model. Note that the lower (measured modes) have the largest influence on the flexibility matrix. The flexibility method (Pandey and Biswas, 1994) compares the flexibility matrices for the healthy and damaged structure, based on the $r$ measured modes, as

$$
\delta \mathbf{C}=\mathbf{C}_{u}-\mathbf{C}_{d}
$$


where

$$
\mathbf{C}_{u}=\sum_{i=1}^{r} \frac{1}{\omega_{u i}^{2}} \phi_{u i} \phi_{u i}^{\top}, \quad \mathbf{C}_{d}=\sum_{i=1}^{r} \frac{1}{\omega_{d i}^{2}} \phi_{d i} \phi_{d i}^{\top} .
$$

A measure in terms of degrees of freedom is obtained by taking the maximum along each column of $\delta \mathbf{C}$.

\section{Sensor Validation}

The correct functioning of structural health monitoring systems requires that the sensors be functioning. Errors introduced by faulty sensors can cause undamaged areas to be identified as damaged. In many civil structures applications for health monitoring (such as bridges), ambient loads must be used for excitation. These loads are not known and may be measured or estimated as part of the health monitoring algorithm, which requires a large number of sensors. Sensor validation, where the sensors are confirmed to be functioning during operation, seems to have received little attention. The critical aspect in structural health monitoring is that there are usually more sensors than excited modes. This redundancy may be used, together with a modal model of the structure, to validate the sensor functionality.

The control and chemical engineering community have considered the sensor validation problem, and have used models and sensor redundancy to good effect. However, these approaches usually use the faulty sensor to predict the response and look for errors between predictions and measurement. Clearly using the faulty sensor in the prediction process will propagate errors to the predicted responses. Often neural networks, or artificial intelligence approaches are used for the analysis.

Friswell and Inman (1999) assumed that only the lower modes of the structure are usually excited, producing a large redundancy in the data. This has similarities to the principal component analysis used in chemical plant (Dunia et al., 1996; Dunia and Qin, 1998). Moreover, the approach seems to work only under the assumption of additive faults while giving erroneous results for the multiplicative faults case. Physically additive faults might arise from DC offsets in the electronic equipment and multiplicative faults might arise from calibration errors. The alternative used here, is to generate new residuals using the modal filtering approach which has similarities to the approach of Friswell and Inman. It is shown that these new residuals have interesting fault isolation properties. The approach is demonstrated on a subframe structure, although the method is completely general and may be applied to any structure for which a modal model is available. If necessary, such a model could be obtained from an identification experiment. For fault isolation a correlation index is proposed which is shown to correctly identify the faulty sensor.

Faults may cause a variety of changes in the dynamic response of a sensor, and many of these are difficult to model. However the two most common faults, namely additive and multiplicative faults, are relatively straightforward to model. Physically additive faults might arise from DC offsets in the electronic equipment and multiplicative faults might arise from calibration errors. In this section the sensor faults are assumed to be additive and modelled as a constant signal added to the sensor response. The problem of 
detecting sensor faults is then transformed into the problem of the detection of the change in the mean of a Gaussian variable with known covariance matrix, which switches from zero under the no-fault condition to a mean value with unknown magnitude under the fault condition. This problem may be solved using a likelihood ratio test resulting in a $\chi^{2}$ distributed variable which is then compared to a threshold. In order to decide which sensor or subset of sensors is most likely to be responsible for the fault, the so-called sensitivity tests are computed, which are also $\chi^{2}$ distributed.

\subsection{Sensor Validation Concepts}

Although there is redundancy in the data, based on the number of sensors and the number of modes excited, it is still not straightforward to identify those sensors that are damaged. When all sensors are working it is possible to estimate the modal contributions to the response and therefore produce a predicted response that will give some idea of the accuracy of the model of structure and the extent of the measurement noise. However if a sensor is damaged, then using data from this sensor to estimate the modal participation factors will propagate the errors from the faulty channel through the estimate of the modal response to the estimate of the response in all channels. Thus to predict faulty sensors the sensors are split into two groups. If $S$ represents the set of all sensors then these two groups are,

$$
\begin{aligned}
& S_{f}=\{\text { sensors assumed to be faulty }\} \\
& S_{w}=\{\text { sensors assumed to be working }\}
\end{aligned}
$$

Note that these two sets are disjoint so that

$$
S_{f} \cap S_{w}=\{\}, \quad S_{f} \cup S_{w}=S .
$$

Note that the distribution of faulty and working sensors seems to have been determined at the outset. In practice which sensors will be faulty is unknown and so every potential subset of faulty sensors must be tried. This approach has parallels with the subset selection technique in parameter estimation (Friswell et al., 1997; Millar, 1990). The difficulty in sensor validation, as in parameter estimation, is to determine which sensor or parameter subset is optimal. Note that for sensor validation, the number of assumed working sensors should be at least as great as the number of modes of interest.

\subsection{Validation via Modal Filtering}

Central to the proposed strategy for sensor validation is a modal model of the structure and also the estimation of the modal participation factors during operation. At any time instant, $t_{k}$, the measured output is

$$
\mathbf{y}\left(t_{k}\right)=\mathbf{y}_{k}=\mathbf{H} \boldsymbol{\Phi} \mathbf{q}\left(t_{k}\right)=\mathbf{H} \boldsymbol{\Phi}_{r} \mathbf{q}_{r}\left(t_{k}\right)+\mathbf{H} \boldsymbol{\Phi}_{d} \mathbf{q}_{d}\left(t_{k}\right)
$$

where the modes have been split into those that are retained, $\boldsymbol{\Phi}_{r}$, and those that will be discarded, $\boldsymbol{\Phi}_{d}$. If $\mathbf{H}_{w}$ picks out those outputs that are assumed to be working (i.e. are elements of $\left.S_{w}\right)$, then we need to estimate $\mathbf{q}_{r, k}=\mathbf{q}_{r}\left(t_{k}\right)$ from

$$
\mathbf{y}_{w, k}=\mathbf{H}_{w} \mathbf{\Phi}_{r} \mathbf{q}_{r, k}+\mathbf{H}_{w} \mathbf{\Phi}_{d} \mathbf{q}_{d, k}
$$


where $\mathbf{y}_{w, k}$ denotes the response at the fully functioning sensors at time $t_{k}$ and $\mathbf{q}_{d, k}=$ $\mathbf{q}_{d}\left(t_{k}\right)$. Clearly the discarded modes in Equation (6.4) could be neglected and the pseudo inverse used to estimate $\mathbf{q}_{r, k}$ from the resulting over-determined set of equations, as

$$
\hat{\mathbf{q}}_{r, k}=\left(\mathbf{H}_{w} \boldsymbol{\Phi}_{r}\right)^{\dagger} \mathbf{y}_{w, k}
$$

where ()$^{\dagger}$ denotes the usual Moore-Penrose pseudo inverse. This gives an estimate of the response at the functioning sensors as

$$
\hat{\mathbf{y}}_{w, k}=\mathbf{H}_{w} \mathbf{\Phi}_{r}\left(\mathbf{H}_{w} \mathbf{\Phi}_{r}\right)^{\dagger} \mathbf{y}_{w, k}=\mathbf{P y}_{w, k}
$$

There will be an error introduced because

$$
\left(\mathbf{H}_{w} \boldsymbol{\Phi}_{r}\right)^{\dagger} \mathbf{H}_{w} \Phi_{d} \neq \mathbf{0}
$$

and a better estimate may be obtained by using the orthogonality of the modes as

$$
\hat{\mathbf{q}}_{r, k}=\left(\boldsymbol{\Phi}_{r}^{\top} \mathbf{H}_{w}^{\top} \mathbf{M}_{w, r} \mathbf{H}_{w} \boldsymbol{\Phi}_{r}\right)^{-1} \boldsymbol{\Phi}_{r}^{T} \mathbf{H}_{w}^{\top} \mathbf{M}_{w, r} \mathbf{y}_{w, k}
$$

where $\mathbf{M}_{w, r}$ is the mass matrix reduced to the degrees of freedom corresponding to the functioning sensors in the set $S_{w}$. Given that the mode shapes are assumed known, SEREP would be the most appropriate reduction method (O'Callahan et al., 1989). However, if the discarded modes lie outside the frequency range of interest then the estimator based on the pseudo inverse, Equation (6.5), will be adequate. The corresponding estimate of the response is

$$
\hat{\mathbf{y}}_{w, k}=\mathbf{H}_{w} \boldsymbol{\Phi}_{r}\left(\boldsymbol{\Phi}_{r}^{\top} \mathbf{H}_{w}^{\top} \mathbf{M}_{w, r} \mathbf{H}_{w} \boldsymbol{\Phi}_{r}\right)^{-1} \boldsymbol{\Phi}_{r}^{\top} \mathbf{H}_{w}^{\top} \mathbf{M}_{w, r} \mathbf{y}_{w, k}=\mathbf{P y}_{w, k} .
$$

Both approaches give a projector matrix $\mathbf{P}$ from the response space to the space of the lower modes. The quality of the model may be determined by reconstructing the response at the functioning sensors and producing the error as

$$
\varepsilon_{w, k}=(\mathbf{I}-\mathbf{P}) \mathbf{y}_{w, k} .
$$

Reconstructing the responses of the faulty sensors gives the error as

$$
\varepsilon_{f, k}=\mathbf{y}_{f, k}-\mathbf{H}_{f} \mathbf{\Phi}_{r} \hat{\mathbf{q}}_{r, k}
$$

where $\mathbf{H}_{f}$ picks out those outputs that are assumed to be faulty (i.e. are elements of $\left.S_{f}\right)$.

In practice we do not know which sensors are working and which are faulty. Therefore the errors in Equations (6.10) and (6.11) are generated for all possible sets $S_{w}$ and $S_{f}$. Of course the estimation of the modal participation factors has been performed at every time step, and so the errors will be produced at every time step. The average error over the time range of interest may be easily computed. The projector matrix, $\mathbf{P}$, is constant for a particular choice of sets $S_{w}$ and $S_{f}$ and only needs to be computed once. Those sets where the error in the faulty sensor(s) is much greater than the error in the functioning sensors are then used to locate the faulty sensors. 


\subsection{The Parity Space Approach}

Abdelghani and Friswell (2001) introduced a different approach to treating the residuals, that performs better on systems with multiplicative sensor errors. Three residuals are required. The first is related to the modal residuals given above, and is essentially the negative of the residual in Equation (6.10), and is

$$
\gamma_{k}=\left[\mathbf{H}_{w} \boldsymbol{\Phi}_{r}\left(\mathbf{H}_{w} \mathbf{\Phi}_{r}\right)^{\dagger}-\mathbf{I}\right] \mathbf{y}_{w, k} .
$$

The second residual is similar, but the complete set of sensors (including any faulty sensor) is used to calculate the modal quantities. Thus

$$
\gamma_{k}^{0}=\mathbf{H}_{w} \boldsymbol{\Phi}_{r}\left(\boldsymbol{\Phi}_{r}\right)^{\dagger} \mathbf{y}_{k}-\mathbf{y}_{w, k} .
$$

The final residual is the difference between the two, namely

$$
\xi_{k}=\gamma_{k}^{0}-\gamma_{k} .
$$

The damage correlation index is then given by

$$
\rho=\frac{E\left[\xi^{\top} \gamma^{0}\right]}{\sqrt{\mathrm{E}\left[\xi^{\top} \xi\right]}}
$$

where the expected value is over the time index $k$. This correlation index may be computed for each potentially faulty sensor, and hence any faulty sensor determined.

\subsection{Example}

The structure considered in this study consists of a suspended steel subframe used extensively in modal identification studies (Abdelghani et al., 1997). The structure was excited at two different locations using random noise inputs, and 28 accelerometers were used to measure the time response. The analysis was performed in the $0-500 \mathrm{~Hz}$ frequency range and 32000 data points per channel were collected at $1024 \mathrm{~Hz}$ sampling frequency.

All 28 sensors were used to identify the experimental natural frequencies, damping ratios and mode shapes from the first 3000 data samples. The Balanced Realization algorithm using data correlations was used for the identification (Abdelghani et al., 1999). Only the 5 first modes were retained (up to $300 \mathrm{~Hz}$ ) and the natural frequencies and damping ratios are given in Table 9. The corresponding real mode shapes were then used to generate the residuals. Faults are added to the measured signals to simulate realistic behaviour. For all cases the data samples 3000-4000 were used to generate the residuals.

Suppose that an additive fault is simulated, where of $50 \%$ of the maximum response is added to sensor 6 . Figure 18 shows the results using modal residuals and demonstrates that additive faults may be detected.

Next a multiplicative fault to sensor 6 is introduced: the time responses are multiplied by a factor of 1.5. The modal residuals perform poorly on multiplicative faults. Figure 19 
Table 9. Frequencies and damping ratios identified using the Balanced Realisation algorithm.

\begin{tabular}{|c|c|}
\hline Frequency $(\mathrm{Hz})$ & Damping (\%) \\
\hline 60.72 & 0.13 \\
156.32 & 0.17 \\
190.66 & 0.16 \\
229.19 & 0.20 \\
287.11 & 0.10 \\
\hline
\end{tabular}

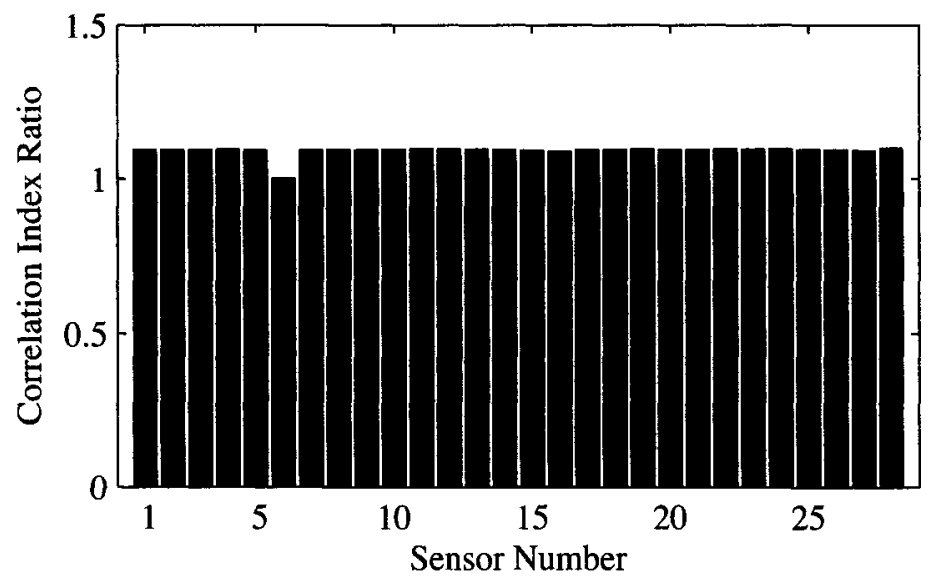

Figure 18. Correlation coefficient ratio under faulty and non-faulty conditions using modal residuals: Sensor 6 has an additive fault.

gives the results using modal residuals and clearly the fault has not been detected. Figure 19 shows the results of the parity space approach and shows that the technique is able to clearly isolate multiplicative faults. Furthermore it seems from these experiments that the neighbouring correlation ratios are not influenced by the faulty sensor(s). Figure 21 shows the results for a complete loss of sensor 8 . Only the ratio of the corresponding sensor changes, while the others stay relatively unchanged. Finally the correlation ratio seems to be related to the amount of damage introduced to the sensors.

All of the above results are based on five modes and 28 sensor locations. As the number of modes used increases, the subspace in which the response lies increases, whereas the subspace in which the errors lie decreases. Thus the performance of the fault location scheme decreases. Similarly using more sensors improves the results, since by increasing the number of sensors the redundancy in the data is increased, which improves the detection and isolation of the faults. As an example a smaller number of sensors was used for the experiment, and more modes were included. Figure 22 shows the results using 10 sensors and nine modes, for the fault at sensor 8 , and should be compared to Figure 21. Fault detection has now failed. 


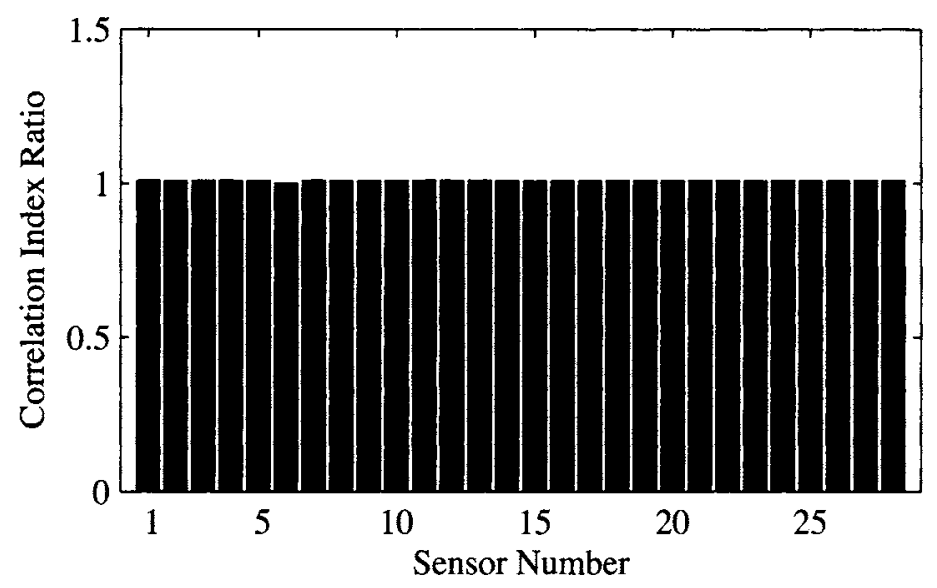

Figure 19. Correlation coefficient ratio under faulty and non-faulty conditions using modal residuals. Sensor 6 has a multiplicative fault.

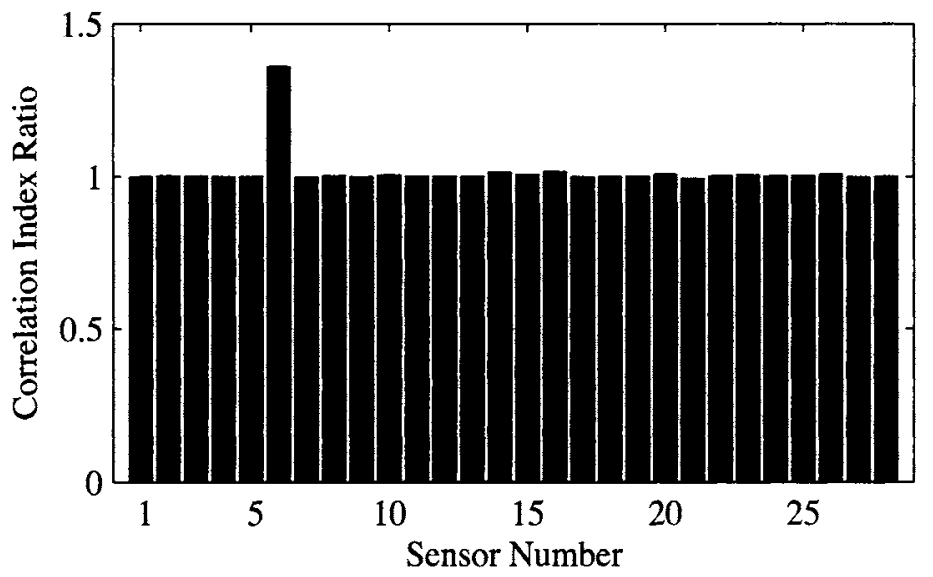

Figure 20. Correlation coefficient ratio under faulty and non-faulty conditions. Sensor 6 has a multiplicative fault. 


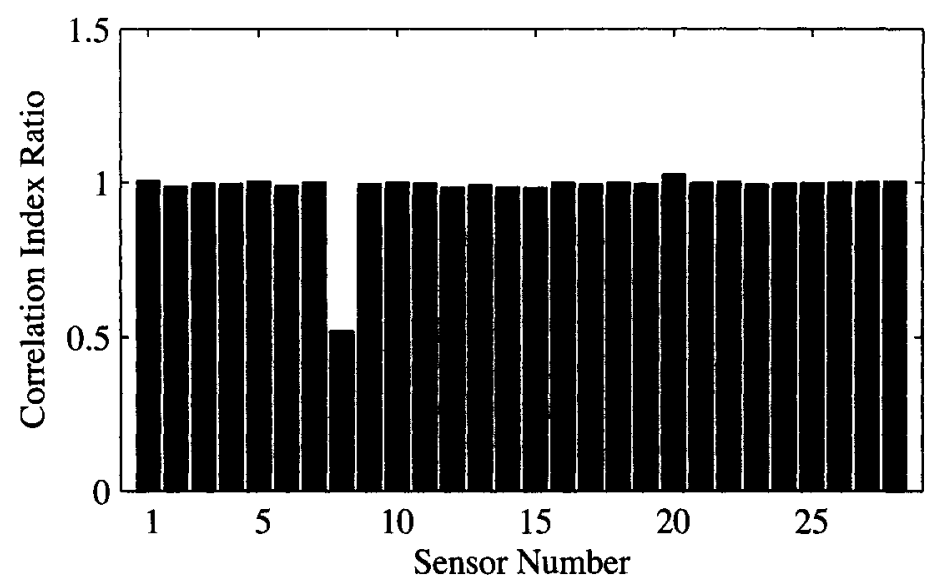

Figure 21. Correlation coefficient ratio under faulty and non-faulty conditions: complete loss of sensor 8 .

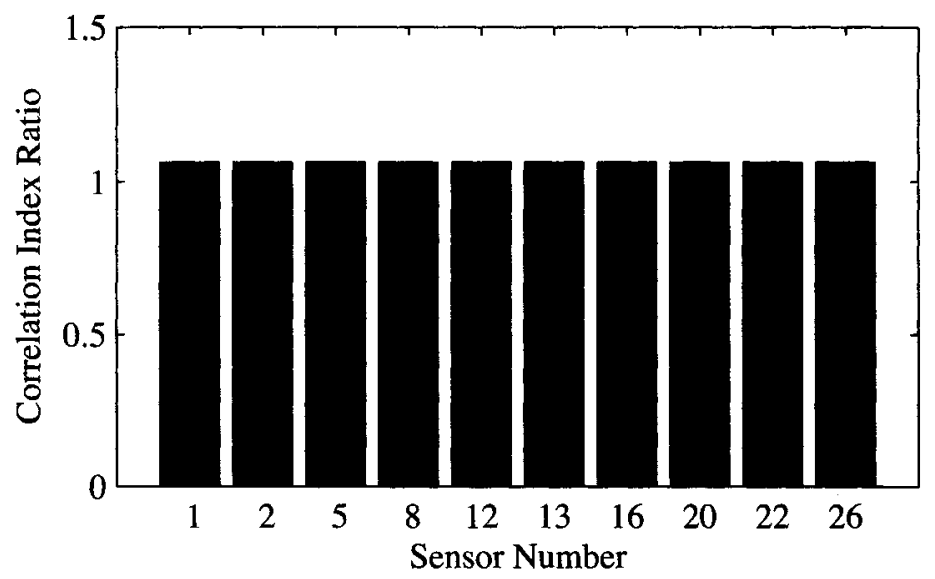

Figure 22. Correlation ratio under faulty and non-faulty conditions: Sensor 8 faulty. Nine modes used for the 10 sensors. 


\section{Conclusions}

This chapter has given a brief introduction to the huge literature available on the approaches of damage identification based on inverse methods. The sensitivity based methods to identify physical parameters using subset selection for error localisation has been suggested as a viable approach. However, many difficulties remain to be fully solved, such as the modelling error between the model and the physical structure, and the influence of environmental factors. The most promising route is to include measurements of temperature, humidity and other environmental variables within the model, although this requires more stringent conditions on modelling error. At the very least these errors give a lower bound on the level of damage that can be detected and localised, and this can be formalised using statistics from the response of the undamaged structure in its normal operating environment. One scenario is that damage location using low frequency vibration is undertaken to identify those areas where more detailed local inspection should be concentrated. The application of robust damage detection and location algorithms based on monitoring the in-service response of a structure remains a challenge, although the availability of a model does open the way to more accurate prognosis and the estimation of the remaining life.

\section{Acknowledgements}

The author gratefully acknowledges the support of the Royal Society through a Royal Society-Wolfson Research Merit Award. The author also acknowledges the contribution of his co-workers, highlighted by reference to published papers throughout this chapter.

\section{Bibliography}

M. O. Abdalla, K. M. Grigoriadis, and D. C. Zimmerman. Enhanced structural damage detection using alternating projection methods. AIAA Journal, 36:1305-1311, 1998.

M. O. Abdalla, K. M. Grigoriadis, and D. C. Zimmerman. Structural damage detection using linear matrix inequality methods. Journal of Vibration and Acoustics, 122 : 448-455, 2000.

M. Abdelghani and M. I. Friswell. A parity space approach to sensor validation. In Proceedings of the 19th International Modal Analysis Conference, pages 405-411, Orlando, Florida, 2001.

M. Abdelghani, C. T. Chou, and M. Verhaegen. Using subspace methods in the identification and modal analysis of structures. In Proceedings of the 15th International Modal Analysis Conference, pages 1392-1398, Orlando, Florida, 1997.

M. Abdelghani, M. Goursat, T. Biolchini, L. Hermans, and H. van der Auweraer. Performance of output-only identification algorithms for modal analysis of aircraft structures. In Proceedings of the 17th International Modal Analysis Conference, pages 224-230, Kissimmee, Florida, 1999.

D. E. Adams and M. Nataraju. A nonlinear dynamical systems framework for structural diagnosis and prognosis. International Journal of Engineering Science, 40:1919-1941, 2002 . 
S. Adhikari and M. I. Friswell. Eigenderivative analysis of asymmetric non-conservative systems. International Journal for Numerical Methods in Engineering, 51:709-733, 2001.

H. Ahmadian, J. E. Mottershead, and M. I. Friswell. Regularisation methods for finite element model updating. Mechanical Systems and Signal Processing, 12:47-64, 1998.

K. J. Atherton, C. A. Paget, and E. W. O'Brien. Structural health monitoring of metal aircraft structures with modified acoustic emission. In Proceedings of SEM X International Conference on Experimental and Applied Mechanics, California, USA, 2004.

E. Balmes. Structural Dynamics Toolbox: For use with MATLAB. Users Guide, Version 4, 2000.

T. Belytschko, Y. Y. Lu, and L. Gu. Crack propagation by element-free Galerkin methods. Engineering Fracture Mechanics, 51:295-315, 1995.

J. A. Brandon. Some insights into the dynamics of defective structures. Proceedings of the Institution of Mechanical Engineers Part C: Journal of Mechanical Engineering Science, 212:441-454, 1998.

S. H. S. Carneiro and D. J. Inman. Comments on the free vibrations of beams with a single-edge crack. Journal of Sound and Vibration, 244:729-736, 2001.

S. H. S. Carneiro and D. J. Inman. Continuous model for the transverse vibration of cracked Timoshenko beams. Journal of Vibration and Acoustics, 124:310-320, 2002.

P. Cawley and R. D. Adams. The location of defects in structures from measurements of natural frequencies. Journal of Strain Analysis, 14:49-57, 1979.

P. Cawley, R. D. Adams, C. J. Pye, and B. J. Stone. A vibration technique for nondestructively assessing the integrity of structures. Journal of Mechanical Engineering Science, 20:93-100, 1978.

S. Christides and A. D. S. Barr. One dimensional theory of cracked Bernoulli-Euler beams. International Journal of Mechanical Science, 26:639-648, 1984.

A. D. Dimarogonas. Vibration of cracked structures: a state of the art review. Engineering Fracture Mechanics, 55:831-857, 1996.

S. W. Doebling. Minimum-rank optimal update of elemental stiffness parameters for structural damage identification. AIAA Journal, 34:2615-2621, 1996.

S. W. Doebling, C. R. Farrar, and M. B. Prime. A summary review of vibration-based damage identification methods. Shock and Vibration Digest, 30:91-105, 1998.

J. V. A. dos Santos, C. M. M. Soares, C. A. M. Soares, and N. M. M. Maia. Structural damage identification in laminated structures using FRF data. Comoposite Structures, 67:239-249, 2005.

R. Dunia and S. J. Qin. Subspace approach to multidimensional fault identification and reconstruction. AIChE Journal, 44:1813-1831, 1998.

R. Dunia, S. J. Qin, T. F. Edgar, and T. J. McAvoy. Identification of faulty sensors using principal component analysis. AIChE Journal, 42:2797-2812, 1996.

C. R. Farrar and D. A. Jauregui. Comparative study of damage identification algorithms applied to a bridge: I. experiment. Smart Materials and Structures, 7:704-719, 1998a.

C. R. Farrar and D. A. Jauregui. Comparative study of damage identification algorithms applied to a bridge: II. numerical study. Smart Materials and Structures, 7:720-731, 1998b. 
M. I. Friswell. Damage identification using inverse methods. Special Issue of the Royal Society Philosophical Transactions on Structural Health Monitoring and Damage Prognosis, 365:393-410, 2007.

M. I. Friswell and D. J. Inman. Sensor validation for smart structures. Journal of Intelligent Material Systems and Structures, 10:973-982, 1999.

M. I. Friswell and J. E. Mottershead. Finite Element Model Updating in Structural Dynamics. Kluwer Academic Publishers, 1995.

M. I. Friswell and J. E. T. Penny. Crack modelling for structural health monitoring. Structural Health Monitoring: An International Journal, 1:139-148, 2002.

M. I. Friswell, J. E. T. Penny, and D. A. Wilson. Using vibration data and statistical measures to locate damage in structures. Modal Analysis: The International Journal of Analytical and Experimental Modal Analysis, 9:239-254, 1994.

M. I. Friswell, J. E. T. Penny, and S. D. Garvey. Parameter subset selection in damage location. Inverse Problems in Engineering, 5:189-215, 1997.

M. I. Friswell, J. E. Mottershead, and H. Ahmadian. Finite element model updating using experimental test data: parameterisation and regularisation. Transactions of the Royal Society of London, Series A, 359:169-186, 2001.

C. P. Fritzen, D. Jennewein, and T. Kiefer. Damage detection based on model updating methods. Mechanical Systems and Signal Processing, 12:163-186, 1998.

G. M. L. Gladwell and H. Ahmadian. Generic element matrices suitable for finite element model updating. Mechanical Systems and Signal Processing, 9:601-614, 1995.

G.H. Golub and C. F. van Loan. Matrix Computations. The John Hopkins University Press, 1996.

P. C. Hansen. Analysis of discrete ill-posed problems by means of the L-curve. SIAM Review, 34:561-580, 1992.

P. C. Hansen. Regularisation tools: a MATLAB package for analysis and solution of discrete ill-posed problems. Numerical Algorithms, 6:1-35, 1994.

F. M. Hemez and C. Farhat. Bypassing the numerical difficulties associated with the updating of finite element matrices. AIA A Journal, 33:539-546, 1995.

K. M. Holford, A. W. Davies, R. Pullin, and D. C. Carter. Damage location in steel bridges by acoustic emission. Journal of Intelligent Material Systems and Structures, $12: 567-576,2001$.

T. J. Johnson, R. L. Brown, D. E. Adams, and M. Schiefer. Distributed structural health monitoring with a smart sensor array. Mechanical Systems and Signal Processing, 18: $555-572,2004$.

A. M. Kabé. Stiffness matrix adjustment using modal data. AIAA Journal, 23:1431-1436, 1985.

T. Y. Kam and T. Y. Lee. Detection of cracks in structures using modal test datas. Engineering Fracture Mechanics, 42:381-387, 1992.

M. Kaouk and D. C. Zimmerman. Structural damage assessment using a generalised minimum rank perturbation theory. AIAA Journal, 32:836-842, 1994.

R. Kenigsbuch and Y. Halevi. Model updating in structural dynamics: A generalised reference basis approach. Mechanical Systems and Signal Processing, 12:75-90, 1998. 
G. Kerschen, J. C. Golinval, and F. M. Hemez. Bayesian model screening for the identification of nonlinear mechanical structures. Journal of Vibration and Acoustics, 125: 389-397, 2003.

M. Kisa and J. A. Brandon. The effects of closure of cracks on the dynamics of a cracked cantilever beam. Journal of Sound and Vibration, 238:1-18, 2000.

A. V. Knyazev and M. E. Argentati. Principal angles between subspaces in an A-based scalar product: algorithms and perturbation estimates. SIAM Journal on Scientific Computing, 23:2008-2040, 2002.

S. S. Law, T. H. T. Chan, and D. Wu. Efficient numerical model for the damage detection of a large scale complex structure. Engineering Structures, 23:436-451, 2001.

Y.-S. Lee and M.-J. Chung. A study on crack detection using eigenfrequency test data. Computers and Structures, 77:327-342, 2001.

N. A. J. Lieven. Spatial correlation of mode shapes, the coordinate modal assurance criterion. In Proceedings of the 6th IMAC, pages 690-695, 1988.

M. Link and M. I. Friswell. Working group 1. generation of validated structural dynamic models - results of a benchmark study utilising the GARTEUR SM-AG19 testbed. Mechanical Systems and Signal Processing, COST Action Special Issue, 17:9-20, 2003.

$\mathrm{H}$. Luo and S. Hanagud. Delamination detection using dynamic characteristics of composite plates. In Proceedings of the AIAA/ASME/ASCE/AHS Structures, Structural Dynamics 8 Materials Conference, pages 129-139, California, USA, 1995.

P. M. Majumdar and S. Suryanarayan. Flexural vibrations of beams with delaminations. Journal of Sound and Vibration, 125:441-461, 1988.

I. W. Mayes and W. G. R. Davies. Analysis of the response of a multi-rotor-bearing system containing a transverse crack in a rotor. Journal of Vibration, Acoustics, Stress and Reliability in Design, 106:139-145, 1984.

S. Meyer and M. Link. Modelling and updating of local non-linearities using frequency response residuals. Mechanical Systems and Signal Processing, 17:219-226, 2003.

T. Mickens, M. Schulz, M. Sundaresan, A.Ghoshal, A. S. Naser, and R. Reichmeider. Structural health monitoring of an aircraft joint. Mechanical Systems and Signal Processing, 17:285-303, 2003.

A. J. Millar. Subset Selection in Regression. Monographs on Statistics and Applied Probability 40, Chapman and Hall, 1990.

J. E. Mottershead and M. I. Friswell. Model updating in structural dynamics: a survey. Journal of Sound and Vibration, 167:347-375, 1993.

J. E. Mottershead, M. I. Friswell, and C. Mares. A method for determining modelstructure errors and for locating damage in vibrating systems. Meccanica, 34:153-166, 1999.

S. A. Neild, M. S. Williams, and P. D. McFadden. Nonlinear vibration characteristics of damaged concrete beams. Journal of Structural Engineering, 129:260-268, 2003.

J. M. Nichols, M. D. Todd, and J. R. Wait. Using state space predictive modeling with chaotic interrogation in detecting joint preload loss in a frame structure. Smart Materials \& Structures, 12:580-601, 2003a. 
J. M. Nichols, L. N. Virgin, M. D. Todd, and J. D. Nichols. On the use of attrator dimension as a feature in structural health monitoring. Mechanical Systems and Signal Processing, 17:1305-1320, 2003b.

J. C. O'Callahan, P. Avitabile, and R. Riemer. System equivalent reduction expansion process (SEREP). In Proceedings of the 7th International Modal Analysis Conference, Las Vegas, pages 29-37, 1989.

K. Ono. Fatigue Crack Measurement: Techniques and Applications, chapter Acoustic emission, pages 173-205. Engineering Materials Advisory Service Ltd., 1991.

W. Ostachowicz and M. Krawczuk. On modelling of structural stiffness loss due to damage. In DAMAS 2001: 4th International Conference on Damage Assessment of Structures, Cardiff, UK, pages 185-199, 2001.

A. K. Pandey and M. Biswas. Damage detection in structures using changes in flexibility. Journal of Sound and Vibration, 169:3-17, 1994.

A. K. Pandey, M. Biswas, and M. M. Samman. Damage detection from changes in curvature mode shapes. Journal of Sound and Vibration, 145:321-332, 1991.

A. Paolozzi and I. Peroni. Detection of debonding damage in a composite plate through natural frequency variations. Journal of Reinforced Plastics and Composites, 9:369$389,1990$.

G. Park, H. Cudney, and D. J. Inman. Impedance-based health monitoring of civil structural components. ASCE Journal of Infrastructure Systems, 6:153-160, 2000.

G. Park, H. Cudney, and D. J. Inman. Feasibility of using impedance-based damage assessment for pipeline systems. Journal of Earthquake Engineering \& Structural Dynamics, 30:1463-1474, 2001.

E. Parloo, P. Guillaume, and M. van Overmeire. Damage assessment using mode shape sensitivities. Mechanical Systems and Signal Processing, 17:499-518, 2003.

B. Peeters and G. de Roeck. One-year monitoring of the Z24-bridge: enviromental effects versus damage events. Earthquake Engineering \& Structural Dynamics, 30:149-171, 2001.

B. N. Rao and S. Rahman. A coupled meshless - finite element method for fracture analysis of cracks. International Journal of Pressure Vessels and Piping, 78:647-657, 2001.

P. F. Rizos, N. Aspragathos, and A. D. Dimarogonas. Identification of crack location and magnitude in a cantilever beam from the vibration modes. Journal of Sound and Vibration, 138:381-388, 1990.

L. M. Rogers. Structural and Engineering Monitoring by Acoustic Emission Methods Fundamentals and Applications. Technical Report, Lloyds Register of Shipping, London, England, 2001.

A. Rytter. Vibration Based Inspection of Civil Engineering Structures. PhD Dissertation, Aalborg University, Denmark, 1993.

M. J. Schulz, M. J. Pai, and D. J. Inman. Health monitoring and active control of composite structures using piezoceramic patches. Composites Part B: Engineering, 30:713-725, 1999. 
C. B. Scruby and D. J. Buttle. Fatigue Crack Measurement: Techniques and Applications, chapter Quantitative fatigue crack measurement by acoustic emission, pages 207-287. Engineering Materials Advisory Service Ltd., 1991.

M.-H. H. Shen. Structronic Systems: Smart Structures, Devices, And Systems, Vol. 1: Smart Materials and Structures, chapter On-line structural damage detection, pages 271-332. World Scientific, 1998.

M.-H. H. Shen and C. Pierre. Free vibration of beams with a single-edge crack. Journal of Sound and Vibration, 170:237-259, 1994.

J. K. Sinha, M. I. Friswell, and S. Edwards. Simplified models for the location of cracks in beam structures using measured vibration data. Journal of Sound and Vibration, 251:13-38, 2002.

H. Sohn, M. Dzwonczyk, E. G. Straser, A.S. Kiremidjian, K. H. Law, and T. Meng. An experimental study of temperature effects on modal parameters of the Alamosa Canyon bridge. Earthquake Engineering \& Structural Dynamics, 28:879-897, 1999.

H. Sohn, C. R. Farrar, N. F. Hunter, and K. Worden. Structural health monitoring using statistical pattern recognition techniques. Journal of Dynamic Systems Measurement and Control, 123:706-711, 2001.

H. Sohn, K. Worden, and C. R. Farrar. Statistical damage classification under changing environmental and operational conditions. Journal of Intelligent Material Systems and Structures, 13:561-574, 2002.

N. Stubbs, J.-T. Kim, and K. G. Topole. An efficient and robust algorithm for damage localization in offshore platforms. In Proceedings of the ASCE 10th Structures Congress, pages 543-546, 1992.

H. Tada, P. Paris, and G. Irwin. The Stress Analysis of Cracks Handbook. Del Research Corporation, 1973.

A. Teughels and G. De Roeck. Structural damage identification of the highway bridge Z24 by FE model updating. Joumal of Sound and Vibration, 278:589-610, 2004.

A. Teughels, J. Maeck, and G. De Roeck. Damage assessment by FE model updating using damage functions. Computers and Structures, 80:1869-1879, 2002.

B. Titurus, M. I. Friswell, and L. Starek. Damage detection using generic elements: part I, model updating. Computers and Structures, 81:2273-2286, 2003a.

B. Titurus, M. I. Friswell, and L. Starek. Damage detection using generic elements: part II, damage detection. Computers and Structures, 81:2287-2299, 2003b.

J. J. Tracy and G. C. Pardoen. Effect of delamination on the natural frequencies of composite laminates. Journal of Composite Materials, 23:1200-1215, 1989.

I. Trendafilova and W. Heylen. Categorisation and pattern recognition methods for damage localisation from vibration measurements. Mechanical Systems and Signal Processing, 17:825-836, 2003.

D. Wang, M. I. Friswell, P.E. Nikravesh, and E. Y. Kuo. Damage detection in structural joints using generic joint elements. In Proceedings of the 17th International Modal Analysis Conference (IMAC), pages 792-798, Orlando, Florida, 1999.

M. G. Wood. Damage Analysis of Bridge Structures using Vibrational Techniques. PhD Thesis, Aston University, UK, 1992. 
K. Worden. Structural fault detection using a novelty measure. Journal of Sound and Vibration, 201:85-101, 1997.

K. Worden, G. Manson, and N. R. J. Fieller. Damage detection using outlier analysis. Journal of Sound and Vibration, 229:647-667, 2000.

L. M. Zhang, Q. Wu, and M. Link. A structural damage identification approach based on element modal strain energy. In Proceedings of ISMA23, pages 107-114, Leuven, Belgium, 1998.

Q. W. Zhang, L. C. Fan, and W. C. Yuan. Traffic induced variability in dynamic properties of cable-stayed bridge. Earthquake Engineering 8 Structural Dynamics, 31: 2015-2021, 2002.

D. C. Zimmerman and M. Kaouk. Structural damage detection using a minimum rank update theory. Journal of Vibration and Acoustics, 116:222-230, 1994.

D. C. Zimmerman, M. Kaouk, and T. Simmermacher. On the role of engineering insight and judgement in structural damage detection. In Proceedings of the 13th International Modal Analysis Conference (IMAC), pages 414-420, Nashville, TN, 1995.

Y. Zou, L. Tong, and G. P. Steven. Vibration-based model-dependent damage (delamination) identification and health monitoring for composite structures - a review. Journal of Sound and Vibration, 230:357-378, 2000. 